\title{
TENSOR PRODUCTS OF COMPOSITION ALGEBRAS, ALBERT FORMS AND SOME EXCEPTIONAL SIMPLE LIE ALGEBRAS
}

\author{
B. N. ALLISON
}

\begin{abstract}
In this paper, we study algebras with involution that are isomorphic after base field extension to the tensor product of two composition algebras. To any such algebra $(\mathscr{A},-)$, we associate a quadratic form $Q$ called the Albert form of $(\mathscr{A},-)$. The Albert form is used to give necessary and sufficient conditions for two such algebras to be isotopic. Using a Lie algebra construction of Kantor, we are then able to give a description of the isomorphism classes of Lie algebras of index $F_{4,1}^{21},{ }^{2} E_{6,1}^{29}, E_{7,1}^{48}$ and $E_{8,1}^{91}$. That description is used to obtain a classification of the indicated Lie algebras over $\mathbf{R}\left(\left(T_{1}, \ldots, T_{n}\right)\right), n \leq 3$.
\end{abstract}

If $\left(\mathscr{C}_{1},-\right)$ and $\left(\mathscr{C}_{2},-\right)$ are composition algebras of dimension $m_{1}$ and $m_{2}$ respectively (with their standard involutions), then we call an algebra with involution that is isomorphic to $\left(\mathscr{C}_{1},-\right) \otimes\left(\mathscr{C}_{2},-\right)$ an $\left(m_{1}, m_{2}\right)$-product algebra or simply a product algebra. Product algebras and their forms (algebras that become product algebras after base field extension) are members of a class of nonassociative algebras with involution called structurable algebras. Hence, given a form $(\mathscr{A},-)$ of a product algebra, a Lie algebra $\mathscr{K}(\mathscr{A},-)$ can be constructed using a construction of Kantor $[\mathbf{2 0}, \mathbf{5}]($ see $\S 1)$.

From the point of view of the study of exceptional simple Lie algebras over nonalgebraically closed fields, the most interesting case occurs when one of the composition algebras has dimension 8 . Indeed, we show in $\S 6$ of this paper that the Kantor construction induces a 1-1 correspondence of the set of isotopy classes of division algebra forms of $(8, m)$-product algebras onto the set of isomorphism classes of central simple Lie algebras of index $I$ (as defined by Tits in [29]), where

$$
I=F_{4,1}^{21}, \quad{ }^{2} E_{6,1}^{29}, \quad E_{7,1}^{48}, \quad \text { or } \quad E_{8,1}^{91}
$$

according as $m=1,2,4$ or 8 . Consequently, it is of interest to classify division algebra forms of $(8, m)$-product algebras up to isotopy.

A form of a product algebra $(\mathscr{A},-)$ is either itself a product algebra or it is obtainable via the corestriction functor from a single composition algebra over a quadratic extension (see §2). In either case, one can define in a natural fashion a quadratic form, called the Albert form of $(\mathscr{A},-)$, on the space $\mathscr{S}=\{s \in$ $\mathscr{A} \mid \bar{s}=-s\}$ of skew-hermitian elements (see $\S 3$ ). For example, in the case when

Received by the editors February 3, 1987. A summary of this work was presented at the Winter Meeting of the Canadian Mathematical Society on December 12, 1987.

1980 Mathematics Subject Classification (1985 Revision). Primary 17A75, 17B25; Secondary $10 \mathrm{C} 01$.

Research supported in part by NSERC Grant A8465. 
$(\mathscr{A},-)=\left(\mathscr{C}_{1},-\right) \otimes\left(\mathscr{C}_{2},-\right)$ is a product algebra, $Q$ is defined by $Q\left(s_{1} \otimes 1+1 \otimes s_{2}\right)=$ $\lambda\left(n_{1}\left(s_{1}\right)-n_{2}\left(s_{2}\right)\right)$, where $n_{i}$ is the norm on $\mathscr{C}_{i}, i=1,2$, and $\lambda \neq 0$ is a fixed constant. If $(\mathscr{A},-)$ is a form of an $(8, m)$-product algebra, then $(\mathscr{A},-)$ is a division algebra if and only if its Albert form is anisotropic (and the center of $\mathscr{A}$ is a field if $m=2$ ) (see $\S 3$ ). Moreover, we show in $\S 5$ that two forms of $(8, m)$-product algebras are isotopic if and only if their Albert forms are similar. This result is obtained using some results on the even Clifford algebra of $Q$ proved in $\S 4$ and a lemma of Seligman regarding isomorphisms of graded Lie algebras.

The results mentioned so far reduce the classification of Lie algebras of index $I$ (as above) to the classification of anisotropic Albert forms up to similarity. In the last part of this paper we use this reduction to study Lie algebras of index $I$ over some special fields. In particular, in $\S 7$, we obtain a classification of Lie algebras of index $I$ over the field $\mathbf{R}\left(\left(T_{1}, T_{2}, \ldots, T_{n}\right)\right)$ of iterated Laurent series in $n$ variables with real coefficients for $n \leq 3$.

Two other constructions that can be shown to yield all Lie algebras of index $I$ have been given in [26, pp. 242-258; 27, Chapters 5 and 6] and in [3] (see also $[\mathbf{1 2}, \mathbf{1 3}])$. Seligman's construction in $[\mathbf{2 6}, \mathbf{2 7}]$ is from a quadratic form subject to restrictions on its Clifford algebra (see $\S 6$ ), while the construction in $[3]$ is from a ternary algebra obtained (at least in some cases) from a pair of composition algebras. The emphasis in the present work is on the specific realization of the quadratic forms needed (for exceptional Lie algebra construction) as Albert forms. The results allow one to use both the theory of quadratic forms and the theory of composition algebras in the study of the Lie algebras of index $I$.

1. The Lie algebra $\mathscr{K}(\mathscr{A},-)$. Throughout the paper, we assume that $k$ is a field of characteristic 0 and that all algebras are finite dimensional over $k$. We also assume that all algebras (except of course Lie algebras) are unital.

If $(\mathscr{A},-)$ is an algebra with involution (by which we mean an antiautomorphism of period 2) over $k$, then we put

$$
\mathscr{S}=\mathscr{S}(\mathscr{A},-)=\{s \in \mathscr{A} \mid \bar{s}=-s\} \quad \text { and } \mathscr{H}=\mathscr{H}(\mathscr{A},-)=\{h \in \mathscr{A} \mid \bar{h}=h\} \text {, }
$$

in which case, $\mathscr{A}=\mathscr{P} \oplus \mathscr{H}$. We denote by $L_{x}$ and $R_{x}$ the operators on $\mathscr{A}$ defined by $L_{x} y=x y$ and $R_{x} y=y x$. We use the notation

$$
[x, y]=x y-y x \quad \text { and } \quad[x, y, z]=(x y) z-x(y z)
$$

for the commutator and associator on $\mathscr{A}$ respectively.

A structurable algebra is an algebra $(\mathscr{A},-)$ with involution such that

$$
\left[V_{x, y}, V_{z, w}\right]=V_{\{x, y, z\}, w}-V_{z,\{y, x, w\}}
$$

for all $x, y, z, w \in \mathscr{A}$, where the operator $V_{x, y}$ and the triple product $\{$,$\} on \mathscr{A}$ are defined by

$$
V_{x, y} z=\{x, y, z\}=(x \bar{y}) z+(z \bar{y}) x-(z \bar{x}) y .
$$

For the rest of this section, we assume that $(\mathscr{A},-)$ is a structurable algebra. We next recall the definition and the relevant properties of the Lie algebra $\mathscr{K}(\mathscr{A},-)$.

First of all, let $\operatorname{Instrl}(\mathscr{A},-)$ be the Lie algebra of endomorphisms of $\mathscr{A}$ spanned by $\left\{V_{x, y} \mid x, y \in \mathscr{A}\right\}$. Then, $L_{\mathscr{Y}} L_{\mathscr{\mathcal { Y }}}$ is an ideal of $\operatorname{Instrl}(\mathscr{A},-)[4, \S 2]$. For $A \in \operatorname{Instrl}(\mathscr{A},-)$, define $A^{\varepsilon}, A^{\delta} \in \operatorname{End}_{\not}(\mathscr{A})$ by

$$
A^{\varepsilon}=A-L_{A 1+\overline{A 1}} \quad \text { and } \quad A^{\delta}=A+R_{\overline{A 1}} .
$$


Then, $A \mapsto A^{\varepsilon}$ is an automorphism of $\operatorname{Instrl}(\mathscr{A},-)$ of period $2,\left.A \mapsto A^{\delta}\right|_{\mathscr{S}}$ is a homomorphism of $\operatorname{Instrl}(\mathscr{A},-)$ into the Lie algebra $\operatorname{End}_{\mathscr{l}}(\mathscr{S})$,

$$
\begin{gathered}
V_{x, y}^{\varepsilon}=-V_{y, x}, \quad\left(L_{s} L_{t}\right)^{\varepsilon}=-L_{t} L_{s}, \\
V_{x, y}^{\delta} s=x(\bar{y} s)+(s y) \bar{x} \quad \text { and } \quad\left(L_{s} L_{t}\right)^{\delta} r=s(t r)+r(t s),
\end{gathered}
$$

for $x, y \in \mathscr{A}, r, s, t \in \mathscr{P}[\mathbf{4}, \S 1 ; \mathbf{5}, \S 1]$.

Next put

$$
\mathscr{K}=\mathscr{K}(\mathscr{A},-)=\tilde{\mathscr{N}} \oplus \operatorname{Instrl}(\mathscr{A},-) \oplus \mathscr{N},
$$

where $\mathscr{N}=\{(x, s) \mid x \in \mathscr{A}, s \in \mathscr{S}\}$ and $\tilde{\mathscr{N}}=\{\tilde{n} \mid n \in \mathscr{N}\}$ is a $k$-isomorphic copy of $\mathscr{N}$. Then, $\mathscr{K}$ is a Lie algebra with subalgebra $\operatorname{Instrl}(\mathscr{A},-)$ and multiplication $[$,$] given by$

$$
\begin{array}{cl}
{[A,(x, s)]=\left(A x, A^{\delta} s\right),} & {\left[A,(x, s)^{\sim}\right]=\left(A^{\varepsilon} x, A^{\varepsilon \delta} s\right)^{\sim},} \\
{[(x, r),(y, s)]=(0, x \bar{y}-y \bar{x}),} & {\left[(x, r)^{\sim},(y, s)^{\sim}\right]=(0, x \bar{y}-y \bar{x})^{\sim},}
\end{array}
$$

and

$$
\left[(x, r),(y, s)^{\sim}\right]=-(s x, 0)^{\sim}+V_{x, y}+L_{r} L_{s}+(r y, 0)
$$

for $A \in \operatorname{Instrl}(\mathscr{A},-), x, y \in \mathscr{A}, r, s \in \mathscr{S}[\mathbf{5}, \S 3]$. Moreover, if we put

$$
\begin{gathered}
\mathscr{K}_{-2}=\left\{(0, s)^{\sim} \mid s \in \mathscr{S}\right\}, \quad \mathscr{K}_{-1}=\left\{(x, 0)^{\sim} \mid x \in \mathscr{A}\right\}, \\
\mathscr{K}_{0}=\operatorname{Instrl}(\mathscr{A},-), \quad \mathscr{K}_{1}=\{(x, 0) \mid x \in \mathscr{A}\}, \\
\mathscr{K}_{2}=\{(0, s) \mid s \in \mathscr{S}\}, \quad \text { and } \mathscr{K}_{i}=\{0\} \quad \text { for }|i|>2,
\end{gathered}
$$

then

$$
\mathscr{K}=\bigoplus \sum_{i \in \mathbf{Z}} \mathscr{K}_{i}=\mathscr{K}_{-2} \oplus \mathscr{K}_{1} \oplus \mathscr{K}_{0} \oplus \mathscr{K}_{1} \oplus \mathscr{K}_{2}
$$

is a $\mathbf{Z}$-graded Lie algebra.

The Lie algebra $\mathscr{K}(\mathscr{A},-)$ is central simple if and only if $(\mathscr{A},-)$ is central simple $[\mathbf{5}, \S 5]$. The central simple Lie algebras that are isomorphic to some $\mathscr{K}(\mathscr{A},-)$ are precisely those of positive relative rank [5, Theorem 10], where the relative rank is the dimension of a maximal split toral subalgebra [26, p. 2]. We are particularly interested in this paper in studying central simple Lie algebras of relative rank 1. These can be constructed from central division algebras.

Indeed, recall that $x \in \mathscr{A}$ is said to be conjugate invertible with conjugate inverse $\hat{x} \in \mathscr{A}$ if $V_{x, \hat{x}}=$ Id (or equivalently $V_{\hat{x}, x}=\mathrm{Id}$ ). In that case, $U_{x} \in \operatorname{End}_{\mathscr{\ell}}(\mathscr{A})$, defined by $U_{x} y=\{x, y, x\}$, is invertible, and $\hat{x}=U_{x}^{-1} x$. If each $x \neq 0 \in \mathscr{A}$ is conjugate invertible, we call $(\mathscr{A},-)$ a division algebra. (If $\mathscr{A}$ is a Jordan algebra and $-=\mathrm{Id}$, or if $(\mathscr{A},-)$ is an alternative algebra with involution, then this notion of division algebra agrees with the classical notion of Jordan division algebra or alternative division algebra respectively $[\mathbf{8}$, p. $132 ; \mathbf{7}$, Corollary 3.6]. The term division algebra is therefore unambiguous in these cases.) We then have

THEOREM 1.2 [6, THEOREM 3.1]. $\mathscr{K}(\mathscr{A},-)$ is central simple of relative rank 1 if and only if $(\mathscr{A},-)$ is a central division algebra. Moreover, any relative rank 1 central simple Lie algebra is isomorphic to some $\mathscr{K}(\mathscr{A},-)$.

In view of the above remarks, it is important to study the isomorphism problem for the Lie algebras $\mathscr{K}(\mathscr{A},-)$. For that purpose, recall that two structurable 
algebras $(\mathscr{A},-)$ and $\left(\mathscr{A}^{\prime},-\right)$ are said to be isotopic (written $(\mathscr{A},-) \sim\left(\mathscr{A}^{\prime},-\right)$ ) if there exists an invertible linear mapping $\alpha: \mathscr{A} \mapsto \mathscr{A}^{\prime}$ such that $\alpha\{x, y, z\}=$ $\{\alpha x, \hat{\alpha} y, \alpha z\}$ for some invertible linear mapping $\hat{\alpha}: \mathscr{A} \mapsto \mathscr{A}^{\prime}$ and all $x, y, z \in \mathscr{A}$. In that case $\hat{\alpha}$ is uniquely determined and $\alpha$ is called an isotopy of $(\mathscr{A},-)$ onto $\left(\mathscr{A}^{\prime},-\right)$. We then have

THEOREM 1.3. The following are equivalent:

(a) $(\mathscr{A},-) \sim\left(\mathscr{A}^{\prime},-\right)$,

(b) $\mathscr{K}(\mathscr{A},-) \cong \mathscr{K}\left(\mathscr{A}^{\prime},-\right)$ as $\mathbf{Z}$-graded Lie algebras.

Moreover, if $(\mathscr{A},-)$ and $\left(\mathscr{A}^{\prime},-\right)$ are central division algebras, then (a) and (b) are equivalent to

(c) $\mathscr{K}(\mathscr{A},-) \cong \mathscr{K}\left(\mathscr{A}^{\prime},-\right)$ as Lie algebras.

ProOF. The equivalence of (a) and (b) is proved in [8, Proposition 12.3]. Suppose then that $(\mathscr{A},-)$ and $\left(\mathscr{A}^{\prime},-\right)$ are central division algebras and that there exists a Lie algebra isomorphism $\phi: \mathscr{K}(\mathscr{A},-) \mapsto \mathscr{K}\left(\mathscr{A}^{\prime},-\right)$. By Theorem 1.2, $\mathscr{K}(\mathscr{A},-)$ and $\mathscr{K}\left(\mathscr{A}^{\prime},-\right)$ have relative rank 1 . Thus, $\mathscr{T}=\ell V_{1,1}$ in $\mathscr{K}(\mathscr{A},-)$ and $\mathscr{T}^{\prime}=\mathscr{k} V_{1,1}$ in $\mathscr{K}\left(\mathscr{A}^{\prime},-\right)$ are maximal split toral subalgebras. But then $\phi(\mathscr{T})$ and $\mathscr{T}^{\prime}$ are maximal split toral subalgebras of $\mathscr{K}\left(\mathscr{A}^{\prime},-\right)$. Hence, there exists an automorphism $\theta$ of $\mathscr{K}\left(\mathscr{A}^{\prime},-\right)$ such that $\theta \phi(\mathscr{T})=\mathscr{T}^{\prime}$ [26, Theorem I.2]. But the eigenvalues of $\operatorname{ad}_{\mathscr{N}\left(\mathscr{H}_{,-}\right)} V_{1,1}$ and $\operatorname{ad}_{\mathscr{H}\left(\mathscr{S}^{\prime},-\right)} V_{1,1}$ are $0, \pm 1$, and possibly \pm 2 . Hence, $\theta \phi\left(V_{1,1}\right)= \pm V_{1,1}$. But there exists an automorphism $\varepsilon$ of $\mathscr{K}\left(\mathscr{A}^{\prime},-\right)$ such that $\varepsilon V_{1,1}=-V_{1,1}[\mathbf{5}, \S 2]$. Replacing $\theta$ by $\varepsilon \theta$ if necessary, we may assume that $\theta \phi\left(V_{1,1}\right)=V_{1,1}$. But then, since $\mathscr{K}_{i}$ is the $i$-eigenspace of $\operatorname{ad}_{\mathscr{K}(\mathscr{A},-)}\left(V_{1,1}\right)$ for $i \in \mathbf{Z}$, it follows that $\theta \phi$ preserves the $\mathbf{Z}$-grading.

As a consequence of the above results, we see that the isomorphism classes of relative rank 1 central simple Lie algebras are in 1-1 correspondence with the isotopy classes of central structurable division algebras. We are therefore interested in the isotopy problem for central simple structurable algebras, and we will pay particular attention to the division algebra case.

2. Forms of product algebras. The class of central simple structurable algebras is made up of five subclasses [4, Theorem 25]. One of the most interesting of these subclasses consists of forms of tensor products of composition algebras. Our goal in this paper is to identify the division algebras and study the isotopy problem in this subclass.

By a composition algebra $(\mathscr{C},-)$, we will mean a composition algebra $\mathscr{C}$ in the usual sense [14] together with its standard involution -. In that case, we denote by $n=n_{\digamma}$ and $t=t_{\digamma}$ the norm and trace respectively on $\mathscr{C}$. Then, $\bar{x}=-x+t(x) 1$ for $x \in \mathscr{C}$, and so

$$
\mathscr{H}(\mathscr{H},-)=\mathscr{\ell} 1 \quad \text { and } \quad \mathscr{P}(\mathscr{C},-)=\{x \in \mathscr{C} \mid t(x)=0\} .
$$

We call the restriction of $n$ to $\mathscr{P}(\mathscr{C},-)$ the pure norm of $\mathscr{C}$. We also denote by $n$ the bilinear form on $\mathscr{C}$ defined by $n(x, y)=n(x+y)-n(x)-n(y)$.

If $\left(\mathscr{C}_{i},-\right)$ is a composition algebra of dimension $m_{i}, i=1,2$, then the algebra $\left(\mathscr{C}_{1},-\right) \otimes\left(\mathscr{C}_{2},-\right)$ (with tensor product multiplication and involution) is structurable $[4, \S 8]$. We call an algebra with involution $(\mathscr{A},-)$ that is isomorphic to such an algebra $\left(\mathscr{C}_{1},-\right) \otimes\left(\mathscr{C}_{2},-\right)$ an $\left(m_{1}, m_{2}\right)$-product algebra or simply a product algebra. 
In that case we can identify

$$
(\mathscr{A},-)=\left(\mathscr{C}_{1},-\right) \otimes\left(\mathscr{C}_{2},-\right) .
$$

We can then identify $\mathscr{C}_{1}$ and $\mathscr{C}_{2}$ with the subalgebras $\mathscr{C}_{1} \otimes 1$ and $1 \otimes \mathscr{C}_{2}$ respectively of $(\mathscr{A},-)$. We then have

$$
\mathscr{S}=\mathscr{S}_{1} \otimes 1 \oplus 1 \otimes \mathscr{S}_{2}=\mathscr{S}_{1} \oplus \mathscr{S}_{2},
$$

where $\mathscr{S}_{i}=\mathscr{S}\left(\mathscr{C}_{i},-\right), i=1,2$. If $\mathscr{S} \neq 0$, the subspaces $\mathscr{S}_{i}$ that are nonzero can be characterized as the maximal subspaces of $\mathscr{S}$ consisting of elements whose squares are in $\ell$. Hence, the subspaces $\mathscr{S}_{i}$ and therefore the subalgebras $\mathscr{C}_{i}$ are determined up to order by $(\mathscr{A},-)$; that is they are independent up to order of the identifying isomorphism. We call the subalgebras $\mathscr{C}_{1}$ and $\mathscr{C}_{2}$ the factors of $(\mathscr{A},-)$.

By a form of an $\left(m_{1}, m_{2}\right)$-product algebra or simply a form of a product algebra, we mean an algebra with involution $(\mathscr{A},-)$ such that $(\mathscr{A},-)_{L}=(\mathscr{A},-) \otimes_{\mathscr{K}} L$ is an $\left(m_{1}, m_{2}\right)$-product algebra over $L$ for some extension $L / k$. We can construct forms of product algebras via the corestriction functor.

Indeed, suppose that $(\mathscr{C},-)$ is a composition algebra of dimension $m>1$ over a quadratic extension $K$ of $h$. Let $\operatorname{Gal}(K / h)=\langle\sigma\rangle$. Let $(\sigma \mathscr{C},-)$ be the composition algebra over $K$ with the same addition, multiplication, and involution as $(\mathscr{C},-)$, but with new scalar multiplication o given by $\alpha \circ x=\sigma(\alpha) x$. Then, let $\operatorname{cor}_{K / \mathscr{\ell}}(\mathscr{C},-)$ be the algebra with involution over $h$ consisting of the fixed points in $(\mathscr{C},-) \otimes_{K}$ $(\sigma \mathscr{C},-)$ of the $\sigma$-semilinear automorphism $U$ determined by $U(x \otimes y)=y \otimes x$. In that case the natural mapping $\operatorname{cor}_{K / \mathscr{k}}(\mathscr{C},-) \otimes_{k} K \mapsto(\mathscr{C},-) \otimes_{K}\left({ }^{\sigma} \mathscr{C},-\right)$ is a $K$ algebra isomorphism, and so $\operatorname{cor}_{K / K}(\mathscr{C},-)$ is a form of an $(m, m)$-product algebra. Thus, $\operatorname{cor}_{K / \mathscr{A}}(\mathscr{C},-)$ is structurable. We call an algebra with involution $(\mathscr{A},-)$ that is isomorphic to such an algebra $\operatorname{cor}_{K / \mathbb{k}}(\mathscr{C},-)$ a twisted $(m, m)$-product algebra or simply a twisted product algebra. In that case, we can identify

$$
(\mathscr{A},-)=\operatorname{cor}_{K / \mathbb{k}}(\mathscr{C},-) \text {, }
$$

and we then have

$$
\mathscr{P}=\left\{s_{1} \otimes 1+1 \otimes s_{1} \mid s_{1} \in \mathscr{P}(\mathscr{C},-)\right\} .
$$

The constructions given above are in fact sufficient to give all forms of product algebras. This follows (at least in the case when one of the factors is octonion) from the classification arguments in [4 and 5]. We indicate now a more straightforward (and standard) argument.

THEOREM 2.1. Suppose $(\mathscr{A},-)$ is a form of an $\left(m_{1}, m_{2}\right)$-product algebra. Then, $(\mathscr{A},-)$ is either an $\left(m_{1}, m_{2}\right)$-product algebra or if $m_{1}=m_{2}>1$ a twisted $\left(m_{1}, m_{1}\right)$-product algebra.

ProOF. By hypothesis, there exists an extension $M / \ell$ such that $(\mathscr{A},-)_{M}=$ $(\mathscr{A},-) \otimes_{\mathscr{A}} M$ is an $\left(m_{1}, m_{2}\right)$-product algebra over $M$. By extending $M$ further we may assume that the factors of $(\mathscr{A},-)_{M}$ are split and hence defined over $k$. Hence, $(\mathscr{A},-)_{M} \cong(\mathscr{B},-)_{M}$ for some $\left(m_{1}, m_{2}\right)$-product algebra $(\mathscr{B},-)$ over $\mathscr{k}$. Thus, $(\mathscr{A},-)_{L} \cong(\mathscr{B},-)_{L}$ for some finite Galois extension $L / \mathscr{k}$. Hence,

$$
(\mathscr{A},-)_{L}=\left(\mathscr{D}_{1},-\right) \otimes_{L}\left(\mathscr{D}_{2},-\right) \text {, }
$$


where $\left(\mathscr{D}_{i},-\right)$ is a composition algebra over $L, i=1,2$. Let $G=\operatorname{Gal}(L / k)$. Then, $G$ acts on $(\mathscr{A},-)_{L}$ by semilinear automorphisms with common fixed set $(\mathscr{A},-)$. If $\eta \in G$, either $\eta$ stabilizes the factors $\mathscr{D}_{i}$ or it exchanges them (since the factors of $(\mathscr{A},-)_{L}$ are determined by $\left.(\mathscr{A},-)_{L}\right)$. If each $\eta \in G$ stabilizes the factors, $(\mathscr{A},-)$ is an $\left(m_{1}, m_{2}\right)$-product algebra. Assume otherwise. Then, $m_{1}=m_{2}>1$ and $H=\left\{\eta \in G \mid \eta\left(\mathscr{D}_{i}\right)=\mathscr{D}_{i}, i=1,2\right\}$ is a subgroup of index 2 in $G$. Let $K$ be the fixed field of $H$ in $L$, in which case $[K: \ell]=2$. Put $\operatorname{Gal}(K / \ell)=\langle\sigma\rangle$. Then,

$$
(\mathscr{A},-)_{K}=\left(\mathscr{C}_{1},-\right) \otimes_{K}\left(\mathscr{C}_{2},-\right) \text {, }
$$

where $\left(\mathscr{C}_{i},-\right)$ is a composition algebra over $K$ of dimension $m_{1}, i=1,2$, and $\sigma\left(\mathscr{C}_{2}\right)=\mathscr{C}_{1}$. Hence, the mapping $(\mathscr{A},-)_{K} \mapsto\left(\mathscr{C}_{1},-\right) \otimes_{K}\left(\sigma \mathscr{C}_{1},-\right)$ determined by $x_{1} \otimes_{K} x_{2} \mapsto x_{1} \otimes_{K} \sigma x_{2}$ is a $K$-algebra isomorphism that maps $(\mathscr{A},-)$ onto $\operatorname{cor}_{K / \mathbb{A}}\left(\mathscr{C}_{1},-\right)$.

REMARKS. (i) If $(\mathscr{A},-)=\operatorname{cor}_{K / \mathscr{k}}(\mathscr{C},-)$ is a twisted $(m, m)$-product algebra then $\mathscr{S}$ does not contain any nonzero elements whose square is in $k 1$. Thus, $(\mathscr{A},-)$ is not also a product algebra.

(ii) Suppose that $(\mathscr{A},-)=\left(\mathscr{C}_{1},-\right) \otimes\left(\mathscr{C}_{2},-\right)$ is a product algebra. Then, the Lie algebra $\mathscr{K}(\mathscr{A},-)$ is isomorphic to one of the Lie algebras constructed using Tits' second Lie algebra construction (see [28 or 24, pp. 121-127]). Indeed, let $\mathscr{J}$ be the Jordan algebra of $3 \times 3$-matrices over $\mathscr{C}_{2}$ satisfying $\gamma^{-1} \bar{X}^{t} \gamma=X$, where $\gamma=\operatorname{diag}(1,-1,1)[\mathbf{1 5}, \S 3.2]$. Then, $\mathscr{K}(\mathscr{A},-) \cong \mathscr{T}\left(\mathscr{C}_{1}, \mathscr{J}\right)$, where $\mathscr{T}\left(\mathscr{C}_{1}, \mathscr{J}\right)=$ $\operatorname{Der}\left(\mathscr{C}_{1}\right) \oplus\left(\mathscr{C}_{1}\right)_{0} \otimes \mathscr{J}_{0} \oplus \operatorname{Der}(\mathscr{J})$ is the Lie algebra constructed using the second Tits' construction from $\mathscr{C}_{1}$ and $\mathscr{J}$. Our proof of this remark makes use of the internal characterization (and its proof), given in [5, §4], of the Lie algebras obtained from the construction $(\mathscr{A},-) \mapsto \mathscr{K}(\mathscr{A},-)$. Since the calculations involved are quite lengthy and since we shall not make use of this remark, we omit the details.

We conclude this section by recording a few of the basic facts about forms of $\left(m_{1}, m_{2}\right)$-product algebras. For that purpose, recall that an invariant form on an álgebra with involution $(\mathscr{A},-)$ is a symmetric bilinear form $\chi$ on $\mathscr{A}$ such that

$$
\chi(\bar{x}, \bar{y})=\chi(x, y) \quad \text { and } \quad \chi(z x, y)=\chi(x, \bar{z} y)
$$

for $x, y, z \in \mathscr{A}[\mathbf{4}, \S 6]$. Also, we denote the nucleus and center of $\mathscr{A}$ by $\operatorname{Nuc}(\mathscr{A})$ and $\operatorname{Cent}(\mathscr{A})$ respectively $[\mathbf{2 4}$, pp. 14-15].

PROPOSITION 2.2. Suppose $(\mathscr{A},-)$ is a form of an $\left(m_{1}, m_{2}\right)$-product algebra. Then

(i) There exists a nondegenerate invariant form $\chi$ on $(\mathscr{A},-)$ such that $\chi(1,1)=$ 4 .

(ii) If $\left(m_{1}, m_{2}\right) \neq(1,1)$, then $\mathscr{S} \cup\left\{s^{2} \mid s \in \mathscr{S}\right\}$ spans $\mathscr{A}$, and so $\mathscr{S}$ generates $\mathscr{A}$ as an algebra without unit element.

(iii)

$$
\operatorname{Nuc}(\mathscr{A})=\left\{\begin{array}{l}
\mathscr{A}, \quad \text { if } m_{1}, m_{2} \leq 4 \\
\text { the } 2 \text { or } 4 \text {-dimensional factor } \\
\quad \text { if }\left\{m_{1}, m_{2}\right\}=\{8,2\} \text { or }\{8,4\} \\
\ell 1, \quad \text { otherwise. }
\end{array}\right.
$$


(iv)

$$
\operatorname{Cent}(\mathscr{A})=\left\{\begin{array}{c}
\mathscr{A}, \quad \text { if } m_{1}, m_{2} \leq 2 \\
\text { the 2-dimensional factor, } \\
\quad \text { if }\left\{m_{1}, m_{2}\right\}=\{8,2\} \text { or }\{4,2\} \\
\mathscr{h} 1, \quad \text { otherwise. }
\end{array}\right.
$$

(v) If $\left(m_{1}, m_{2}\right) \neq(2,2)$, then $(\mathscr{A},-)$ is central simple.

(vi) $(\mathscr{A},-)$ is semisimple. (See $[\mathbf{2 5}, \S 2]$.

PROOF. We use standard facts about composition algebras. Namely, the norm bilinear form is invariant and nondegenerate. Also, if $(\mathscr{O},-)$ is an octonion algebra, then $\operatorname{Nuc}(\mathscr{O})=\ell 1, \mathscr{O}$ is central simple, and $\mathscr{S}(\mathscr{O},-)=[\mathscr{S}(\mathscr{O},-), \mathscr{S}(\mathscr{O},-)$, $\mathscr{S}(\mathscr{O},-)]$.

(i) If $(\mathscr{A},-)=\left(\mathscr{C}_{1}^{\prime},-\right) \otimes\left(\mathscr{C}_{2},-\right)$ is a product algebra, then there exists a unique symmetric bilinear form $\chi$ on $\mathscr{A}$ such that $\chi\left(x_{1} \otimes x_{2}, y_{1} \otimes y_{2}\right)=n_{1}\left(x_{1}, y_{1}\right) n_{2}\left(x_{2}, y_{2}\right)$. One easily checks that $\chi$ has the required properties. Suppose then that $(\mathscr{A},-)=$ $\operatorname{cor}_{K / \mathscr{A}}(\mathscr{E},-)$ is a twisted product algebra. Define $\chi$ on $(\mathscr{C},-) \otimes_{K}(\sigma \mathscr{C},-)$ as above. Now $(\mathscr{A},-)$ is spanned by elements of the form $x \otimes x$ and we have $\chi(x \otimes x, y \otimes y)=$ $n_{\mathscr{f}}(x, y) \sigma\left(n_{\mathscr{\digamma}}(x, y)\right) \in \mathscr{K}$, since the norm of $\sigma \mathscr{C}$ is $\sigma \circ n_{\mathscr{C}}$. Thus, $\left.\chi\right|_{\mathscr{A} \times \mathscr{A}}$ does the job.

(ii) It suffices to prove that $\mathscr{H}$ is spanned by elements of the form $s \circ t=s t+t s$, where $s, t \in \mathscr{P}$. For this we can extend the base field and assume $(\mathscr{A},-)=$ $\left(\mathscr{C}_{1},-\right) \otimes\left(\mathscr{C}_{2},-\right)$ is a product algebra. (ii) is then straightforward since $\left(s_{1}+s_{2}\right)^{2}=$ $\left(-n_{1}\left(s_{1}\right)-n_{2}\left(s_{2}\right)\right) 1+2 s_{1} \otimes s_{2}$ for $s_{i} \in \mathscr{S}_{i}$ and $\mathscr{H}=\ell 1 \oplus \mathscr{S}_{1} \otimes \mathscr{S}_{2}$.

In the proofs of (iii) (vi), we can extend the base field and assume $\ell$ is algebraically closed and $(\mathscr{A},-)=\left(\mathscr{C}_{1},-\right) \otimes\left(\mathscr{C}_{2},-\right)$, where $\operatorname{dim}\left(\mathscr{C}_{i}\right)=m_{i}, i=1,2$, and $m_{1} \geq m_{2}$.

(iii) This is clear if $m_{1}, m_{2} \leq 4$. Suppose then that $m_{1}=8$. Since $\mathscr{C}_{2} \subseteq$ $\operatorname{Nuc}(\mathscr{A})$ if $m_{2}=1,2$ or 4 , and $\operatorname{since} \operatorname{Nuc}\left(\mathscr{C}_{2}\right)=\ell 1$ if $m_{2}=8$, it suffices to show that $\operatorname{Nuc}(\mathscr{A}) \subseteq \mathscr{C}_{2}$. Now $\operatorname{Nuc}(\mathscr{A})$ is stabilized by - , and so $\operatorname{Nuc}(\mathscr{A})=(\mathscr{H} \cap$ $\operatorname{Nuc}(\mathscr{A})) \oplus(\mathscr{S} \cap \operatorname{Nuc}(\mathscr{A}))$. But if $h \in \mathscr{H} \cap \operatorname{Nuc}(\mathscr{A})$, we have $\chi(h,[\mathscr{S}, \mathscr{S}, \mathscr{H}])=$ $\chi([\mathscr{S}, \mathscr{S}, h], \mathscr{H})=\{0\}$. Also $\mathscr{S}_{1} \otimes \mathscr{S}_{2}=\left[\mathscr{S}_{1}, \mathscr{S}_{1}, \mathscr{S}_{1}\right] \otimes \mathscr{S}_{2}=\left[\mathscr{S}_{1}, \mathscr{S}_{1}, \mathscr{S}_{1} \otimes \mathscr{S}_{2}\right] \subseteq$ $[\mathscr{S}, \mathscr{S}, \mathscr{H}]$. Hence, $\chi\left(h, \mathscr{S}_{1} \otimes \mathscr{S}_{2}\right)=\{0\}$. On the other hand, $\chi(h, \mathscr{S})=\{0\}$. But the same argument shows that $\ell 1$ is the orthogonal complement of $\left(\mathscr{S}_{1} \otimes \mathscr{S}_{2}\right) \oplus \mathscr{S}$ with respect to $\chi$. Hence, $\mathscr{H} \cap \operatorname{Nuc}(\mathscr{A}) \subseteq \mathscr{l} 1$. But $\operatorname{Nuc}(\mathscr{A})$ is a subalgebra and so this forces $\operatorname{Nuc}(\mathscr{A}) \subseteq \mathscr{C}_{1}$ or $\mathscr{C}_{2}$. Since $\operatorname{Nuc}\left(\mathscr{C}_{1}\right)=\ell 1$, we have $\operatorname{Nuc}(\mathscr{A}) \subseteq \mathscr{C}_{2}$.

(iv) This follows from (iii) and standard associative facts.

(v) It suffices to show that $(\mathscr{A},-)$ is simple. This is clear if $\left(m_{1}, m_{2}\right)=(1,1)$ or $(2,1)$. Thus, we may assume that $m_{1} \geq 4$. Suppose that $\mathscr{I}$ is a proper ideal of $(\mathscr{A},-)$. Then, $\mathscr{I}=(\mathscr{I} \cap \mathscr{H}) \oplus(\mathscr{I} \cap \mathscr{S})$. Now if $\mathscr{I}$ contains a nonzero element of $\mathscr{S}_{i}$, then $\mathscr{I}$ contains $\mathscr{C}_{i}$ and $\mathscr{I}=\mathscr{A}$. Thus, $\mathscr{I} \cap \mathscr{S}_{i}=\{0\}, i=1,2$. Thus, if $s=s_{1}+s_{2} \neq 0 \in \mathscr{I} \cap \mathscr{S}$, where $s_{i} \in \mathscr{S}_{i}, i=1,2$, we must have $s_{1}, s_{2} \neq 0$. But then there exists $t_{1} \in \mathscr{S}_{1}$ so that $\left[s_{1}, t_{1}\right] \neq 0$ and so $\left[s, t_{1}\right]=\left[s_{1}, t_{1}\right] \neq 0 \in \mathscr{I} \cap \mathscr{S}_{1}$. This is a contradiction and therefore $\mathscr{I} \subseteq \mathscr{H}$. Finally, let $h \in \mathscr{I}$. Then, for $s \in \mathscr{S}$, $h s+s h \in \mathscr{I} \cap \mathscr{P}$ and so $h s+s h=0$. Hence, $\chi\left(h, s^{2}\right)=-\frac{1}{2} \chi(s h+h s, s)=0$ for $s \in \mathscr{S}$. Thus, $h=0$. 
(vi) This follows from (v) if $\left(m_{1}, m_{2}\right) \neq(2,2)$. Suppose that $\left(m_{1}, m_{2}\right)=(2,2)$. Then, $(\mathscr{A},-) \cong(h \oplus h, \mathrm{ex}) \otimes(h \oplus h, \mathrm{ex}) \cong(h \oplus h, \mathrm{ex}) \oplus(h \oplus h, \mathrm{ex})$, where ex is the exchange involution, and so $(\mathscr{A},-)$ is semisimple.

3. The Albert form $Q$ and the paper that $(\mathscr{A},-)$ is a form of an $\left(m_{1}, m_{2}\right)$-product algebra.

The key to the study of $(\mathscr{A},-)$ is a quadratic form $Q=Q_{(\mathscr{A},-)}$ defined on $\mathscr{P}=\mathscr{P}(\mathscr{A},-)$ called the Albert form of $(\mathscr{A},-)$. In the case when $\left(m_{1}, m_{2}\right)=$ $(4,4)$, this form has been used by Albert [1] and Jacobson [18] in their studies of $(4,4)$-product algebras. The terminology follows Jacobson [18]. We also introduce a linear mapping $\downarrow=\natural_{(, \cdots,-)}: \mathscr{S} \mapsto \mathscr{S}$, called the $\downarrow$-mapping of $(\mathscr{A},-)$, that is closely related to $Q$. To define $Q$ and $\hbar$ we consider cases.

Suppose first of all that $(\mathscr{A},-)=\left(\mathscr{C}_{1},-\right) \otimes\left(\mathscr{C}_{2},-\right)$ is a product algebra. Fix a nonzero constant $\lambda \in \mathscr{R}$. Define $Q: \mathscr{P} \mapsto R$ by

$$
Q\left(s_{1}+s_{2}\right)=\lambda\left(n_{1}\left(s_{1}\right)-n_{2}\left(s_{2}\right)\right)
$$

for $s_{i} \in \mathscr{F}_{i}, i=1,2$, where $n_{i}=n_{\digamma_{i}}, i=1,2 . Q$ is a nondegenerate quadratic form on $\mathscr{P}$. Define $\llbracket: \mathcal{P} \mapsto \mathscr{P}$ by

$$
\left(s_{1}+s_{2}\right)^{\natural}=\lambda\left(s_{1}-s_{2}\right) \text {. }
$$

Since the factors of $(\mathscr{A},-)$ are determined up to order by $(\mathscr{A},-)$, the Albert form $Q$ and the $h$-mapping are determined up to nonzero multiple by $(\mathscr{A},-)$.

Suppose secondly that $(\mathscr{A},-)=\operatorname{cor}_{K / \mathscr{A}}(\mathscr{C},-)$ is a twisted product algebra. Fix a nonzero element $\lambda \in K$ such that $\sigma(\lambda)=-\lambda$, where $\operatorname{Gal}(K / \ell)=\langle\sigma\rangle$. Define $Q: \mathscr{P} \mapsto \mathscr{k}$ by

$$
Q\left(s_{1} \otimes 1+1 \otimes s_{1}\right)=\lambda\left(n\left(s_{1}\right)-\sigma\left(n\left(s_{1}\right)\right)\right)=\operatorname{tr}_{K / k}\left(\lambda n\left(s_{1}\right)\right)
$$

for $s_{1} \in \mathscr{P}(\mathscr{C},-)$, where $\operatorname{tr}_{K / \mathbb{K}}: K \mapsto \mathscr{k}$ is the trace of the extension $K / \mathcal{k}$ and $n=n$. In the language of quadratic forms, $Q$ is the transfer of the pure norm of $\mathscr{C}[\mathbf{2 1}, \S$ VII.1]. Also, define $\measuredangle: \mathscr{P} \mapsto \mathscr{P}$ by

$$
\left(s_{1} \otimes 1+1 \otimes s_{1}\right)^{\natural}=\lambda\left(s_{1} \otimes 1-1 \otimes s_{1}\right)=\left(\lambda s_{1}\right) \otimes 1+1 \otimes\left(\lambda s_{1}\right) .
$$

Observe that the extension of $Q$ and $\natural$ to $(\mathscr{C},-) \otimes_{K}(\sigma \mathscr{C},-)$ are respectively the

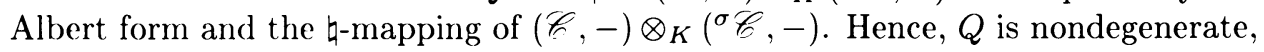
and $Q$ and $\downarrow$ are determined up to nonzero multiple by $(\mathscr{A},-)$.

We have thus defined $Q$ and $\square$ for any form of a product algebra. As usual, we also denote by $Q$ the bilinear form $Q: \mathscr{P} \times \mathscr{P} \mapsto h$ defined by

$$
Q(s, t)=Q(s+t)-Q(s)-Q(t) .
$$

In the rest of this paper, we will use all of the above notation, as well as that of $\S 2$, without comment.

We now want to derive the basic identities satisfied by $Q$ and $\downarrow$. To do so we require the identities

$$
[s, x, y]=-[x, s, y]=[x, y, s]
$$

and the Moufang identity

$$
s(t(s x))=(s t s) x
$$

which hold for $s, t \in \mathscr{P}, x, y \in \mathscr{A}$ in any structurable algebras $(\mathscr{A},-)[\mathbf{4}$, equations (6) and (43)]. (Of course, in (3.2) (and elsewhere), sts can be written unambiguously because of $(3.1)$. 
PROPOSITION 3.3. Suppose $(\mathscr{A},-)$ is a form of a product algebra. Then,

$$
\begin{gathered}
Q\left(s^{\natural}\right)=\lambda^{2} Q(s), \quad\left(s^{\natural}\right)^{\natural}=\lambda^{2} s, \\
s s^{\natural}=s^{\natural} s=-Q(s) 1, \\
L_{s} L_{s^{\natural}}=L_{s^{\natural}} L_{s}=-Q(s) \mathrm{Id}, \\
s t^{\natural} s=Q(s) t-Q(s, t) s, \\
L_{s} L_{t^{\natural}} L_{s}=Q(s) L_{t}-Q(s, t) L_{s}, \\
\left(L_{s} L_{r^{\natural}}\right)\left(L_{s} L_{t^{\natural}}\right)=Q(s) L_{r} L_{t^{\natural}}-Q(s, r) L_{s} L_{t^{\natural}},
\end{gathered}
$$

and

$$
\left(s t^{\natural}\right)^{2}+Q(s, t) s t^{\natural}+Q(s) Q(t) 1=0
$$

for $r, s, t \in \mathcal{P}$.

PROOF. Equations (3.4) are clear. To prove the others, we may extend the base field and assume that $(\mathscr{A},-)=\left(\mathscr{C}_{1},-\right) \otimes\left(\mathscr{C}_{2},-\right)$ and hence that $\lambda=1$. Write $s=s_{1}+s_{2}, t=t_{1}+t_{2}$, where $s_{i}, t_{i} \in \mathscr{S}, i=1,2$. Then, if $x=x_{1} \otimes x_{2}$, where $x_{i} \in \mathscr{C}_{i}, i=1,2$, we have

$$
\begin{aligned}
L_{s} L_{s^{z}} x & =\left(s_{1}+s_{2}\right)\left(\left(s_{1}-s_{2}\right) x_{1} \otimes x_{2}\right)=\left(s_{1}+s_{2}\right)\left(s_{1} x_{1} \otimes x_{2}-x_{1} \otimes s_{2} x_{2}\right) \\
& =\left(s_{1}^{2} x_{1} \otimes x_{2}-x_{1} \otimes s_{2}^{2} x_{2}\right)=\left(-n_{1}\left(s_{1}\right)+n_{2}\left(s_{2}\right)\right) x_{1} \otimes x_{2}=-Q(s) x .
\end{aligned}
$$

Thus, $L_{s} L_{s}=-Q(s)$ Id. Similarly, $L_{s^{\natural}} L_{s}=-Q(s)$ Id. This proves (3.6). Applying (3.6) to 1 yields (3.5). Next

$$
\begin{aligned}
s t^{\natural} s & =\left(s_{1}+s_{2}\right)\left(t_{1}-t_{2}\right)\left(s_{1}+s_{2}\right)=\left(s_{1}+s_{2}\right)\left(t_{1} s_{1}+t_{1} s_{2}-t_{2} s_{1}-t_{2} s_{2}\right) \\
& =s_{1} t_{1} s_{1}-s_{2} t_{2} s_{2}-s_{1}\left(s_{2} t_{2}+t_{2} s_{2}\right)+\left(s_{1} t_{1}+t_{1} s_{1}\right) s_{2}-s_{1}^{2} t_{2}+t_{1} s_{2}^{2} .
\end{aligned}
$$

But $s_{i} t_{i}+t_{i} s_{i}=-n_{i}\left(s_{i}, t_{i}\right) 1$ and

$$
s_{i} t_{i} s_{i}=-t_{i} s_{i}^{2}-n_{i}\left(s_{i}, t_{i}\right) s_{i}=n_{i}\left(s_{i}\right) t_{i}-n_{i}\left(s_{i}, t_{i}\right) s_{i},
$$

$i=1,2$. Thus

$$
\begin{aligned}
s t^{\natural} s= & n_{1}\left(s_{1}\right) t_{1}-n_{1}\left(s_{1}, t_{1}\right) s_{1}-n_{2}\left(s_{2}\right) t_{2}+n_{2}\left(s_{2}, t_{2}\right) s_{2} \\
& +n_{2}\left(s_{2}, t_{2}\right) s_{1}-n_{1}\left(s_{1}, t_{1}\right) s_{2}+n_{1}\left(s_{1}\right) t_{2}-n_{2}\left(s_{2}\right) t_{1} \\
= & \left(n_{1}\left(s_{1}\right)-n_{2}\left(s_{2}\right)\right)\left(t_{1}+t_{2}\right)-\left(n_{1}\left(s_{1}, t_{1}\right)-n_{2}\left(s_{2}, t_{2}\right)\right)\left(s_{1}+s_{2}\right) \\
= & Q(s) t-Q(s, t) s .
\end{aligned}
$$

Thus, we have (3.7). (3.8) follows from (3.7) and (3.2). (3.9) follows from (3.8). Finally,

$$
[s, t, s t]=-[s, s t, t]=-\left(s^{2} t\right) t+s\left(s t^{2}\right)=-s^{2} t^{2}+s^{2} t^{2}=0
$$

and so $(s t)^{2}=s(t(s t))=(s t s) t$. Thus,

$$
\begin{aligned}
\left(s t^{\natural}\right)^{2} & =\left(s t^{\natural} s\right) t^{\natural}=(Q(s) t-Q(s, t) s) t^{\natural} \quad(\text { by }(3.7)) \\
& =-Q(s) Q(t) 1-Q(s, t) s t^{\natural} \quad(\text { by }(3.5))
\end{aligned}
$$

and so we have $(3.10)$.

REMARK. If $\operatorname{dim}(\mathscr{A})>1$, the formulas of Proposition 3.3 have a Jordan theoretic interpretation. Indeed, if we fix $s_{0} \in \mathscr{F}^{\prime}$ such that $Q\left(s_{0}\right) \neq 0$, we can normalize 
$Q$ so that $Q\left(s_{0}\right)=1$. In that case, by (3.6) and (3.9), $\left(L_{s} L_{s_{0}^{\natural}}\right)^{2}+Q\left(s, s_{0}\right) L_{s} L_{s_{0}^{a}}+$ $Q(s)$ Id $=0$. Thus, the mapping $s \mapsto-L_{s} L_{s_{0}^{:}}$is an isomorphism of the Jordan algebra of the quadratic form $Q$ with basepoint [19] onto a subalgebra of $\operatorname{End}_{\mathscr{k}}(\mathscr{A})$ (with its usual Jordan product).

If $s \in \mathscr{F}$, then, according to [8, Proposition 11.1], $s$ is conjugate invertible $\Leftrightarrow L_{s}$ is invertible,

in which case

$$
\hat{s}=-L_{s}^{-1} 1 .
$$

This observation combined with (3.6) yields:

Corollary 3.13. Suppose $s \in \mathscr{P}$. Then,

$$
s \text { is conjugate invertible } \Leftrightarrow Q(s) \neq 0,
$$

in which case $\hat{s}=(1 / Q(s)) s^{\natural}$.

In [6. Theorems 5.1 and 5.2], conditions were given for forms of product algebras to be division algebras. It is important for our purposes, and simpler, to formulate the conditions in terms of the Albert form.

THEOREM 3.14. Let $(\mathscr{A},-)$ be a form of an $\left(m_{1}, m_{2}\right)$-product algebra. Then, $(\mathscr{A},-)$ is a division algebra if and only if the Albert form of $(\mathscr{A},-)$ is anisotropic and cent $(\mathscr{A})$ is a field.

PROOF. If $\left(m_{1}, m_{2}\right)=(1,1)$ or $(2,2)$, then $\operatorname{cent}(\mathscr{A})=\mathscr{A}$ and the theorem follows from Corollary 3.13. Suppose that $\left(m_{1}, m_{2}\right) \neq(1,1)$ or $(2,2)$. Then, $(\mathscr{A},-)$ is central simple and $\mathscr{A}$ is generated, as an algebra without unit element, by $\mathscr{S}$. But then according to [6, Theorem 3.2], $(\mathscr{A},-)$ is a division algebra if and only if $\operatorname{Cent}(\mathscr{A})$ is a field and every nonzero element of $\mathscr{S}$ is conjugate invertible. The result then follows from Corollary 3.13.

REMARK. The factors of a product division algebra are division algebras, since subalgebras of division algebras are division algebras [6, §2]. Also, if a twisted product algebra $\operatorname{cor}_{K / \mathscr{K}}(\mathscr{C},-)$ is a division algebra, then $(\mathscr{C},-)$ is a division algebra, since otherwise there exists $s_{1} \in \mathscr{P}(\mathscr{C},-)$ such that $n\left(s_{1}\right)=-1$ and so

$$
Q\left(s_{1} \otimes 1+1 \otimes s_{1}\right)=0 .
$$

COROllary 3.15. Suppose $(\mathscr{A},-)$ is a twisted $(m, m)$-product algebra. Then, $(\mathscr{A},-)$ is a division algebra if and only if the Albert form of $(\mathscr{A},-)$ is anisotropic.

PROOF. This follows from Theorem 3.14 and Proposition 2.2(iv) if $m=4$ or 8. Suppose then that $m=2$ and that $Q$ is anisotropic. Now $\mathscr{A}$ is the direct sum of fields, $\operatorname{dim}(\mathscr{A})=4$, and - is an automorphism of $\mathscr{A}$ with $\operatorname{dim}(\mathscr{H}(\mathscr{A},-))=2$. Thus, either $\mathscr{A}$ is a field, $(\mathscr{A},-) \cong(\mathscr{D},-) \oplus(\mathscr{E},-)$, where $(\mathscr{D},-),(\mathscr{E},-)$ are 2-dimensional composition algebras, or $(\mathscr{A},-) \cong(L \oplus L$, ex $)$, where $L / \mathcal{R}$ is a quadratic extension and ex is the exchange involution. In the second case there exists $s \neq 0 \in \mathcal{F}$ such that $L_{s}$ is not invertible, and so $Q$ is not anisotropic. In the last case, there exists $s \neq 0 \in \mathscr{P}$ such that $s^{2} \in \ell$, and so $(\mathscr{A},-)$ is not twisted. Thus, $\mathscr{A}$ is a field. 
4. Instrl $(\mathscr{A},-)$ and the even Clifford algebra of $Q$. In this section, we study the structure of the Lie algebras $\mathscr{K}_{0}=\operatorname{Instrl}(\mathscr{A},-)$ as well as the (related) structure of the even Clifford algebra of $Q$.

If $q$ is quadratic form defined on a vector space $\mathscr{V}$, we denote by $C(\mathscr{V}, q)$ (resp. $\left.C^{+}(\mathscr{V}, q)\right)$ the Clifford algebra (resp. the even Clifford algebra) of $q$. We will make frequent use of the classical structure theorem for $C^{+}(\mathscr{V}, Q)$ [17, Theorem 4.13]. We also require the following universal property:

LEMMA 4.1. Suppose $q$ is a nondegenerate quadratic form on a vector space $\mathscr{V}$. Suppose that $\phi: \mathscr{V} \times \mathscr{V} \mapsto \mathscr{B}$ is a bilinear mapping into an associative algebra $\mathscr{B}$ such that

$$
\phi(u, v) \phi(u, w)=-q(u) \phi(v, w)+q(u, v) \phi(u, w) \quad \text { and } \quad \phi(u, u)=q(u) 1
$$

for $u, v \in \mathscr{V}$. Then, there exists a unique algebra homomorphism $\theta: C^{+}(\mathscr{V}, q) \mapsto$ $\mathscr{B}$ such that $\theta(u v)=\phi(u, v)$ for $u, v \in \mathscr{V}$.

Proof. The uniqueness of $\theta$ is clear. We may assume $\mathscr{V} \neq\{0\}$. Fix $z \in \mathscr{V}$ with $q(z) \neq 0$. Let $\mathscr{Y}=(k z)^{\perp}$, with respect to $q$. Now if $y \in \mathscr{Y}$,

$$
\phi(z, y)^{2}=-q(z) \phi(y, y)=-q(z) q(y) 1 .
$$

Hence, there exists an algebra homomorphism $\theta_{1}: C(\mathscr{Y},-q(z) q) \mapsto \mathscr{B}$ such that $\theta_{1}(y)=\phi(z, y), y \in \mathscr{Y}$. But there exists an isomorphism $\theta_{2}: C(\mathscr{Y},-q(z) q) \mapsto$ $C^{+}(\mathscr{V}, q)$ such that $\theta_{2}(y)=z y, y \in \mathscr{Y}\left[\mathbf{1 7}\right.$, p. 236]. Put $\theta=\theta_{1} \circ \theta_{2}^{-1}$. Then, $\theta(z z)=\theta(q(z) 1)=q(z) 1=\phi(z, z)$. Also if $y \in \mathscr{Y}$, then $\theta(z y)=\theta_{1}(y)=\phi(z, y)$ and so $\theta(y z)=-\theta(z y)=-\phi(z, y)$. But linearizing $\phi(u, u)=q(u) 1$ gives $\phi(u, v)+$ $\phi(v, u)=q(u, v) 1$, and so $\phi(z, y)=-\phi(y, z)$ for $y \in \mathscr{Y}$. Thus, $\theta(y z)=\phi(y, z)$ for $y \in \mathscr{Y}$. Finally, if $w, y \in \mathscr{Y}$,

$$
\begin{aligned}
q(z) \theta(y w) & =\theta\left(y z^{2} w\right)=-\theta(z y z w)=-\theta(z y) \theta(z w) \\
& =-\phi(z, y) \phi(z, w)=q(z) \phi(y, w)-q(z, y) \phi(z, w)=q(z) \phi(y, w)
\end{aligned}
$$

and so $\theta(y w)=\phi(y, w)$.

PROPOSITION 4.2. There exists a unique algebra homomorphism $\theta: C^{+}(\mathscr{S}, Q)$ $\mapsto \operatorname{End}_{k^{\prime}}(\mathscr{A})$ such that $\theta\left(s_{C} t\right)=-L_{s} L_{t^{\natural}}$, for $s, t \in \mathscr{S}$, where the product $s_{C} t$ on the left is in $C(\mathscr{S}, Q)$.

ProOF. This follows immediately from (3.6), (3.9), and Lemma 4.1.

From now on $\theta$ will denote the homomorphism of Proposition 4.2. For $s, t \in \mathscr{S}$, define $M_{s, t} \in \operatorname{End}_{\not}(\mathscr{A})$ by

$$
M_{s, t}=L_{s} L_{t^{\natural}}-L_{t} L_{s^{\natural}}
$$

Proposition 4.3. Suppose $\operatorname{dim}(\mathscr{A})>1$. Then, $M_{\mathscr{P}, \mathcal{Y}}$ is an ideal of the Lie algebra $L, L_{,}$,

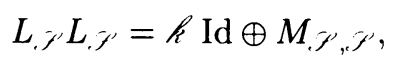

and the mapping $A \mapsto A^{\delta} \mid, \gamma$ is an isomorphism of $M_{\mathscr{Y}, \mathcal{Y}}$ onto the orthogonal Lie algebra o $(\mathscr{P}, Q)$ of $Q$.

ProOF. By Proposition 4.2, $\theta \mid \mathscr{S}_{C} \mathscr{S}: \mathscr{S}_{C} \mathscr{P} \mapsto L_{\mathscr{Y}} L_{\mathscr{Y}}$ is a surjective Lie algebra homomorphism. But

$$
\mathscr{H}_{C}, \mathscr{P}=h 1 \oplus[\mathscr{P}, \mathscr{P}]_{C},
$$


where $[s, t]_{C}=s_{C} t-t_{C} s$. Hence, $M_{, \mathcal{\gamma}^{\prime}, \mathcal{Y}}$ is an ideal of $L_{\mathscr{\gamma}} L_{\mathscr{f}}$ and

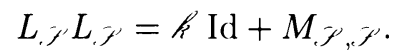

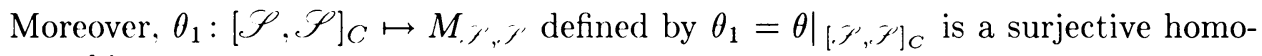
morphism.

Next if $r, s, t \in \mathscr{P}$, then

$$
\begin{aligned}
M_{s, t}^{\delta} r & =s\left(t^{\natural} r\right)+r\left(t^{\natural} s\right)-t\left(s^{\natural} r\right)-r\left(s^{\natural} t\right) \quad(\text { by }(1.1)) \\
& =Q(s, r) t-Q(s, t) r-Q(r, t) s-Q(t, r) s+Q(t, s) r+Q(r, s) t
\end{aligned}
$$

(by the linearization of $(3.7)$ )

$$
=2 Q(s, r) t-2 Q(t, r) s
$$

Thus.

$$
\left.M_{s, t}^{\delta}\right|_{, j}=W_{s, t},
$$

where $W_{s, t} r=2 Q(s, r) t-2 Q(t, r) s$. But the endomorphisms $W_{s, t} \operatorname{span} o(\mathscr{P}, Q)$ [16, p. 232]. Thus, $\theta_{2}: M_{\varkappa}, \mapsto \circ(\mathscr{P}, Q)$ defined by $\theta_{2}(A)=A^{\delta} \mid$., is a surjective homomorphism such that $\theta_{2}\left(M_{s, t}\right)=W_{s, t}$.

But then $\theta_{2} \circ \theta_{1}:[\mathscr{P}, \mathscr{S}]_{C} \mapsto o(\mathscr{P}, Q)$ is a surjective homomorphism. Also

$$
\operatorname{dim}\left([\mathscr{P}, \mathscr{P}]_{C}\right)=\operatorname{dim}(o(\mathscr{P}, Q))=\left(\begin{array}{l}
d \\
2
\end{array}\right)
$$

where $d=\operatorname{dim}(\mathscr{P})\left[\mathbf{1 6}\right.$, p. 232]. Hence, $\theta_{1}$ and $\theta_{2}$ are isomorphisms.

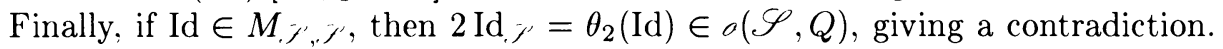
Thus, our decomposition is direct.

We put $\Lambda=$ Nuc $\mathscr{A}$. Then, $\Lambda$ is described in Proposition 2.2(iii). Also, $(\Lambda,-)$ is a subalgebra of $(\mathscr{A},-)$ and so $\mathscr{P}(\Lambda,-)=\mathscr{P} \cap \Lambda$.

TheOREM 4.4. Suppose $\operatorname{dim}(\mathscr{A})>1$. Then, $\operatorname{Instrl}(\mathscr{A},-)=R_{\mathscr{P} \cap \Lambda} \oplus L_{\mathscr{P}} L_{\mathscr{P}}$ as Lie algebras.

PROOF. By Proposition 2.2, $(\mathscr{A},-)$ possesses a nondegenerate invariant bilinear form and $\mathscr{A}$ is generated, as an algebra without 1 , by $\mathscr{P}$. These are the only conditions required to use the argument on pp. 145-146 of [6] that shows that $R_{y^{\prime} \cap \Lambda}$ is the centralizer of $\mathscr{K}_{-2} \oplus L_{y}, L_{y} \oplus \mathscr{K}_{2}$ in $\mathscr{K}$. It follows that $R_{\mathscr{P} \cap \Lambda}$ is the orthogonal complement of $L_{\mathscr{\gamma}} L_{\mathscr{y}}$ in $\mathscr{K}_{0}$, with respect to the Killing form on $\mathscr{K}$. ( $\mathscr{K}$ is semisimple since $(\mathscr{A},-)$ is semisimple $[\mathbf{2 5}, \S 2]$.) Consequently, it suffices to show that $R_{y \cap \Lambda} \cap\left(L_{y}, L_{, y}\right)=\{0\}$. Suppose then that $R_{S} \in R_{\mathscr{P} \cap \Lambda} \cap\left(L_{\mathscr{P}} L_{\mathscr{S}}\right)$.

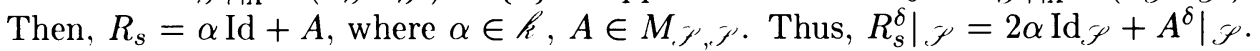
But $\left.R_{s}^{\delta}\right|_{, j}=0$ and $A^{\delta} \mid, \mathcal{\gamma} \in o(\mathcal{P}, Q)$. Taking traces of both sides then gives $\alpha=0$ and hence $A^{\delta} \mid, y=\{0\}$. Thus, $A=0$.

Now $\mathscr{A}$ is a right $\Lambda$-module under the natural action $(a, \lambda) \mapsto a \lambda$. Then, $\operatorname{End}_{\Lambda} \mathscr{A}$ denotes the $\mathscr{R}$-algebra of $\Lambda$-module endomorphisms of $\mathscr{A}$. Also, we use the notation $S^{\dagger}$ to denote the subalgebra (with unit element) generated by a subset $S$ of $\operatorname{End}_{\not} \mathscr{A}$.

THEOREM 4.5. Suppose $(\mathscr{A},-)$ is a form of an $\left(m_{1}, m_{2}\right)$-product algebra and that $\left\{m_{1}, m_{2}\right\} \neq\{1,1\},\{2,1\}$, or $\{2,2\}$. Then,

$$
\left(L, L, H^{\dagger}\right)^{\dagger}=\operatorname{End}_{\Lambda} \mathscr{A} \text {. }
$$


Consequently, $\theta$ is a surjective homomorphism of $C^{+}(\mathscr{S}, Q)$ onto $\operatorname{End}_{\Lambda} \mathscr{A}$. Moreover, $\theta$ is not injective if and only if $\left\{m_{1}, m_{2}\right\}=\{4,4\},\{8,4\}$, or $\{8,8\}$.

Proof. Now $\theta$ maps $C^{+}(\mathscr{S}, Q)$ onto $\left(L_{\mathscr{H}} L_{\mathscr{f}}\right)^{\dagger}$, and we certainly have

$$
\left(L, L, L^{\dagger} \subseteq \operatorname{End}_{\Lambda} \mathscr{A}\right. \text {. }
$$

Thus, it suffices to prove the reverse inclusion and the final statement about the injectivity of $\theta$. For this we may assume that $\ell$ is algebraically closed and that $(\mathscr{A},-)=\left(\mathscr{C}_{1},-\right) \otimes\left(\mathscr{C}_{2},-\right)$, where $\operatorname{dim}\left(\mathscr{C}_{i}\right)=m_{i}, i=1,2$ and $m_{1} \geq m_{2}$. We put $d=\operatorname{dim}(\mathscr{S})=m_{1}+m_{2}-2$ and $e=\operatorname{dim}(\Lambda)$.

Suppose first that $\Lambda$ is simple. Then, $\left(m_{1}, m_{2}\right)=(4,1),(4,4),(8,1),(8,4)$, or $(8,8)$. Now $\operatorname{dim}\left(C^{+}(\mathscr{S}, Q)\right)=2^{d-1}$, and so $\operatorname{dim}\left(\left(L_{\mathcal{P}} L_{\mathscr{P}}\right)^{\dagger}\right)=2^{d-1}$ or $2^{d-2}$ according as $\theta$ is or is not injective. Moreover, $\theta$ must be injective when $d$ is odd (since in that case $C^{+}(\mathscr{P}, Q)$ is simple). Also, since $\Lambda$ is simple, $\operatorname{dim}\left(\operatorname{End}_{\Lambda} \mathscr{A}\right)=\left(m_{1} m_{2}\right)^{2} / e$ (by [17, Theorem 4.11]). Thus, we have the following table of dimensions:

\begin{tabular}{l|ccccc}
$\left(m_{1}, m_{2}\right)$ & $(4,1)$ & $(4,4)$ & $(8,1)$ & $(8,4)$ & $(8,8)$ \\
\hline $\operatorname{dim}\left(\left(L_{j}, L_{\varkappa^{\prime}}\right)^{\dagger}\right)$ & $2^{2}$ & $2^{5}$ or $2^{4}$ & $2^{6}$ & $2^{9}$ or $2^{8}$ & $2^{13}$ or $2^{12}$ \\
$\operatorname{dim}\left(\operatorname{End}_{\Lambda} \mathscr{A}\right)$ & $2^{2}$ & $2^{4}$ & $2^{6}$ & $2^{8}$ & $2^{12}$
\end{tabular}

where the second choice on the second line is correct if and only if $\theta$ is not injective. Then, for $\Lambda$ simple, the result follows from this table and (4.7).

Suppose next that $\Lambda$ is not simple. Then, $\left(m_{1}, m_{2}\right)=(4,2)$ or $(8,2)$, and $\Lambda=\mathscr{A}$ or $\mathscr{C}_{2}$ accordingly. In the first case, End $\mathscr{A}_{\Lambda}=L_{\mathscr{A}}$, while in the second, $\operatorname{End}_{\Lambda} \mathscr{A} \cong$ End $_{\not}, \mathscr{C}_{1} \oplus \operatorname{End}_{\not} \mathscr{C}_{1}$. Thus, $\operatorname{dim}\left(\operatorname{End}_{\Lambda} \mathscr{A}\right)=2^{3}$ or $2^{7}$, and $\operatorname{dim}\left(C^{+}(\mathscr{S}, Q)\right)=2^{3}$ or $2^{7}$ accordingly. Hence, it suffices to show that $\theta$ is injective. For this it suffices to show that $\theta$ is injective on the center of $C^{+}(\mathscr{S}, Q)$. Now we may choose an $n_{1}$-orthogonal basis $s_{1}, \ldots, s_{d-1}$ for $\mathscr{P}_{1}$. Also we may choose $t \in \mathscr{S}_{2}$ such that $n_{2}(t) \neq 0$. Putting $z=\left(s_{1}\right)_{C}\left(s_{2}\right)_{C} \cdots\left(s_{d-1}\right)_{C} t$, the center of $C^{+}(\mathscr{S}, Q)$ is $\ell 1 \oplus k z$ $[\mathbf{1 7}$, p. 237]. But

$$
\theta(z)=L_{s_{1}} L_{s_{2}^{z}} \cdots L_{s_{d-1}} L_{t^{\sharp}}=\alpha L_{s_{1}} L_{s_{2}} \cdots L_{s_{d-1}} L_{t},
$$

where $\alpha \neq 0 \in k$. Consequently, if $\theta$ is not injective, we have $\theta(z) \in \ell$ Id and hence $L_{t}=-n_{2}(t)^{-1} L_{t}^{-1} \in\left(L_{\text {, }}\right)^{d-1}$. But this is impossible, since $L_{t} \mathscr{C}_{1} \nsubseteq \mathscr{C}_{1}$.

COROllary 4.8. Suppose $(\mathscr{A},-)$ is a form of an $\left(m_{1}, m_{2}\right)$-product algebra. Then,

$$
C^{+}(\mathscr{P}, Q) \cong\left\{\begin{array}{l}
\mathscr{H}(\mathscr{A},-), \quad \text { if } m_{1}, m_{2} \leq 2, \\
\operatorname{End}_{\Lambda} \mathscr{A} \oplus \operatorname{End}_{\Lambda} \mathscr{A}, \quad \text { if }\left\{m_{1}, m_{2}\right\}=\{4,4\},\{8,4\} \text { or }\{8,8\}, \\
\operatorname{End}_{\Lambda} \mathscr{A}, \quad \text { otherwise. }
\end{array}\right.
$$

ProOF. Since $C^{+}(\mathscr{S}, Q)$ is either simple or the direct sum of two isomorphic simple ideals [17, p. 237], this follows from the theorem unless $m_{1}, m_{2} \leq 2$. But if $m_{1}, m_{2} \leq 2$, then $\theta$ maps $C^{+}(\mathscr{S}, Q)$ onto $\left(L_{\mathscr{P}} L_{\mathscr{H}}\right)^{\dagger}=L_{\mathscr{H}}^{\dagger}=L_{\mathscr{P}}$. Counting dimensions, we see that $\theta$ is an isomorphism.

5. The isotopy problem. We suppose in this section that $(\mathscr{A},-)$ is a form of an $\left(m_{1}, m_{2}\right)$-product algebra and $\left(\mathscr{A}^{\prime},-\right)$ is a form of an $\left(m_{1}^{\prime}, m_{2}^{\prime}\right)$-product algebra. We wish to solve the isotopy problem for forms of product algebras, that is give 
necessary and sufficient conditions for $(\mathscr{A},-)$ to be isotopic to $\left(\mathscr{A}^{\prime},-\right)$. Now if $(\mathscr{A},-) \sim\left(\mathscr{A}^{\prime},-\right)$, then $\operatorname{dim}(\mathscr{P}(\mathscr{A},-))=\operatorname{dim}\left(\mathscr{P}\left(\mathscr{A}^{\prime},-\right)\right)$ [8, Lemma 12.1] and hence $\left\{m_{1}, m_{2}\right\}=\left\{m_{1},{ }^{\prime}, m_{2}^{\prime}\right\}$. Thus, we may as well assume from the outset that $m_{1}=m_{1}^{\prime}$ and $m_{2}=m_{2}^{\prime}$. We write $\mathscr{P}^{\prime}=\mathscr{P}\left(\mathscr{A}^{\prime},-\right), \mathscr{H}^{\prime}=\left(\mathscr{A}^{\prime},-\right), Q^{\prime}=Q_{\left(\mathscr{H}^{\prime},-\right)}$, etc. For notational convenience, we use $\mathrm{t}_{\text {for }}$ the

If $\left\{m_{1}, m_{2}\right\} \neq\{8,4\}$ or $\{8,8\}$, then the isotopy problem has been studied in [7]. The results there imply

THEOREM 5.1. Suppose that $(\mathscr{A},-)$ and $\left(\mathscr{A}^{\prime},-\right)$ are forms of $\left(m_{1}, m_{2}\right)$ product algebras, where $\left\{m_{1}, m_{2}\right\} \neq\{8,4\}$ or $\{8,8\}$. Then,

$$
(\mathscr{A},-) \sim\left(\mathscr{A}^{\prime},-\right) \Leftrightarrow(\mathscr{A},-) \cong\left(\mathscr{A}^{\prime},-\right) .
$$

Moreover, if $(\mathscr{A},-)$ and $\left(\mathscr{A}^{\prime},-\right)$ are product algebras, then they are isotopic if and only if they have isomorphic factors (up to order).

PROOF. We need only prove the first statement. If $\left(m_{1}, m_{2}\right) \neq(2,2)$, then $(\mathscr{A},-)$ and $\left(\mathscr{A}^{\prime},-\right)$ are simple and alternative, and the result follows from [7, Corollary 7.6]. If $\left(m_{1}, m_{2}\right)=(2,2)$, then $(\mathscr{A} .-)$ and $\left(\mathscr{A}^{\prime},-\right)$ are associative and the result follows from [7, Corollary 4.10].

This theorem is a satisfactory answer to the isotopy problem in the cases covered by its hypotheses. It leaves us with the cases $\left\{m_{1}, m_{2}\right\}=\{8,4\}$ and $\{8,8\}$ to consider. In those cases, we will see that isotopy is equivalent to similarity of the Albert forms. Since the Albert form is of interest in all cases, we do not restrict ourselves only to the consideration of the cases $\left\{m_{1}, m_{2}\right\}=\{8,4\}$ and $\{8,8\}$.

In $q$ and $q^{\prime}$ are quadratic forms on vector spaces $\mathscr{V}$ and $\mathscr{V}^{\prime}$ respectively, recall that a similarity of $q$ onto $q^{\prime}$ is a $\mathscr{k}$-linear bijection $\beta: \mathscr{V} \mapsto \mathscr{V}^{\prime}$ such that $q^{\prime}(\beta v)=$ $\mu q(v)$ for all $v \in \mathscr{V}$ and some fixed nonzero constant $\mu \in \ell$. In that case, we say that $q$ and $q^{\prime}$ are similar and write $q \sim q^{\prime} . \mu$ is called the multiplier of $\beta$.

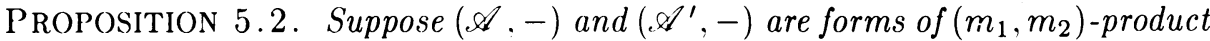
algebras with Albert forms $Q$ and $Q^{\prime}$ respectively. If $(\mathscr{A},-) \sim\left(\mathscr{A}^{\prime},-\right)$, then $Q \sim$ $Q^{\prime}$.

PROOF. Suppose that $\alpha$ is an isotopy of $(\mathscr{A},-)$ onto $\left(\mathscr{A}^{\prime},-\right)$. Then, by $[8$, Lemma 12.1], there exist linear bijections $\beta, \varsigma: \mathscr{P} \mapsto \mathscr{S}^{\prime}$ such that

$$
\alpha L_{s}=L_{\beta s} \hat{\alpha} \text { and } \hat{\alpha} L_{s}=L_{\varsigma s} \alpha
$$

for $s \in \mathcal{P}$. But then if $s \in \mathcal{P}$ is conjugate invertible, we have $\alpha=-\alpha L_{s} L_{\hat{s}}$ (by [8, Theorem 11.4]) $=-L_{\beta s} \hat{\alpha} L_{\hat{s}}=-L_{\beta s} L_{\zeta \hat{s}} \alpha$. Thus $L_{\beta s} L_{\zeta \hat{s}}=-$ Id and so $\beta s$ is conjugate invertible (by (3.11)). Moreover, by (3.12),

$$
(\beta s)^{\wedge}=-L_{\beta s}^{-1} 1=L_{\varsigma \hat{s}} 1=\zeta \hat{s} .
$$

Thus, by Corollary 3.13 , we have

$$
\frac{1}{Q^{\prime}(\beta s)}(\beta s)^{\natural}=\frac{1}{Q(s)} \varsigma\left(s^{\natural}\right)
$$

for $s \in \mathcal{P}$ such that $Q(s) \neq 0$. But by Theorem 5.1, we can assume that $\left\{m_{1}, m_{2}\right\} \neq\{1,1\},\{2,1\}$ or $\{2,2\}$, and hence that $\operatorname{dim}(\mathscr{P})=\operatorname{dim}\left(\mathcal{P}^{\prime}\right) \geq 3$. Thus, $Q$ and $Q^{\prime}$ are irreducible and so $(5.3)$ forces $Q^{\prime}(\beta s)=\mu Q(s)$ for some $\mu \neq 0 \in \mathscr{h}$.

We are now interested in investigating the extent to which the converse of Proposition 5.2 holds. The remainder of this section will be devoted to proving the following. 
THEOREM 5.4. Suppose that $(\mathscr{A},-)$ and $\left(\mathscr{A}^{\prime},-\right)$ are forms of $\left(m_{1}, m_{2}\right)$ product algebras with Albert forms $Q$ and $Q^{\prime}$ respectively. Then

(i) If $\left\{m_{1}, m_{2}\right\}=\{1,1\},\{2,1\}$ or $\{2,2\}$, then

$$
Q \sim Q^{\prime} \Leftrightarrow \mathscr{H} \cong \mathscr{H}^{\prime} .
$$

(ii) If $\left\{m_{1}, m_{2}\right\}=\{4,1\},\{4,2\}$ or $\{4,4\}$, then

$$
Q \sim Q^{\prime} \Leftrightarrow \mathscr{A} \cong \mathscr{A}^{\prime} \text {. }
$$

(iii) If $\left\{m_{1}, m_{2}\right\}=\{8,1\},\{8,2\},\{8,4\}$ or $\{8,8\}$, then

$$
Q \sim Q^{\prime} \Leftrightarrow(\mathscr{A},-) \sim\left(\mathscr{A}^{\prime},-\right) \text {. }
$$

REMARK. If $(\mathscr{A},-)$ and $\left(\mathscr{A}^{\prime},-\right)$ are $(4,4)$-product algebras, this result is proved by Jacobson in $[\mathbf{1 8}$, Theorem $3.12(\mathrm{i})]$. We give a different (although related) proof that includes that case.

Our proof of Theorem 5.4 consists of a sequence of lemmas.

LEMMA 5.5. The implications " $\Leftarrow$ " in Theorem 5.4 are valid.

ProOF. Suppose first that $m_{1}, m_{2} \leq 2$ and that $\mathscr{H}(\mathscr{A},-) \cong \mathscr{H}\left(\mathscr{A}^{\prime},-\right)$. The implication " $\Leftarrow$ " is trivial if $\left\{m_{1}, m_{2}\right\}=\{1,1\}$ or $\{2,1\}$, and so we can assume that $\left\{m_{1}, m_{2}\right\}=\{2,2\}$. Now by Corollary $4.8, C^{+}(\mathscr{S}, Q) \cong C^{+}\left(\mathscr{S}^{\prime}, Q^{\prime}\right)$. But $\operatorname{dim}(\mathscr{S})=\operatorname{dim}\left(\mathscr{P}^{\prime}\right)=2$ and hence, by [17, p. 237], the bilinear forms $Q$ and $Q^{\prime}$ have the same discriminant (mod squares). This forces $Q \sim Q^{\prime}$ (again since $\left.\operatorname{dim}(\mathscr{P})=\operatorname{dim}\left(\mathscr{P}^{\prime}\right)=2\right)$.

Suppose next that $\left\{m_{1}, m_{2}\right\}=\{4,1\},\{4,2\}$, or $\{4,4\}$ and that $\mathscr{A} \cong \mathscr{A}^{\prime}$. If $\left\{m_{1}, m_{2}\right\}=\{4,1\}$, then any isomorphism preserves the involutions, and so $(\mathscr{A},-) \cong\left(\mathscr{A}^{\prime},-\right)$, which implies that $Q \sim Q^{\prime}$. If $\left\{m_{1}, m_{2}\right\}=\{4,2\}$ and the 2 dimensional factor $(=\operatorname{Cent}(\mathscr{A}))$ of $(\mathscr{A},-)$ is split, then $(\mathscr{A},-) \cong\left(\mathscr{D} \oplus \mathscr{D}^{\text {op }}\right.$, ex $)$ and $\left(\mathscr{A}^{\prime},-\right) \cong\left(\mathscr{D}^{\prime} \oplus \mathscr{D}^{\mathrm{op}}, \mathrm{ex}\right)$, where $\mathscr{D}$ and $\mathscr{D}^{\prime}$ are quaternion algebras with opposite algebras $\mathscr{D}^{\mathrm{op}}$ and $\mathscr{J}^{\text {op }}$ respectively, and ex is the exchange involution in each case. But then since $\mathscr{D} \cong \mathscr{D}$ op and $\mathscr{D}^{\prime} \cong \mathscr{D}^{\prime \mathrm{op}}, \mathscr{A} \cong \mathscr{A}^{\prime}$ implies that $\mathscr{D} \cong \mathscr{D}^{\prime}$. Thus, $(\mathscr{A},-) \cong\left(\mathscr{A}^{\prime},-\right)$, which implies that $Q \sim Q^{\prime}$. Thus, we may assume that: (a) $(\mathscr{A},-)$ is a $(4,2)$-product algebra with $\operatorname{Cent}(\mathscr{A})$ a field, or (b) $(\mathscr{A},-)$ is a form of a $(4,4)$-product algebra. Let $\tau$ and $\tau^{\prime}$ denote the involutions on $\mathscr{A}$ and $\mathscr{A}^{\prime}$ respectively. Using the given isomorphism we may identify $\mathscr{A}$ and $\mathscr{A}^{\prime}$ (but not $\tau$ and $\tau^{\prime}$ ). In case (a), $\tau$ and $\tau^{\prime}$ are involutions of the second kind on $\mathscr{A}$. In case (b), $\tau$ and $\tau^{\prime}$ are orthogonal involutions on $\mathscr{A}$, that is $\operatorname{dim}(\mathscr{P}(\mathscr{A}, \tau))=\operatorname{dim}\left(\mathscr{P}\left(\mathscr{A}, \tau^{\prime}\right)\right)=6$. In either case it is well known (and easy to verify) that there exists an invertible $a \in \mathscr{A}$ so that $\tau a=a$ and

$$
\tau^{\prime} x=a(\tau x) a^{-1}
$$

for $x \in \mathscr{A}$. In that case,

$$
\mathscr{P}^{\prime}=\mathscr{P}\left(\mathscr{A}, \tau^{\prime}\right)=a \mathscr{P}(\mathscr{A}, \tau)=a \mathscr{S}
$$

Now replacing $t$ by $t^{\natural}$ in (3.10), we have (by (3.4))

$$
\lambda^{4}(s t)^{2}+\lambda^{2} Q\left(s, t^{\natural}\right) s t+\lambda^{2} Q(s) Q(t) 1=0
$$

for $s, t \in \mathscr{P}$. Similarly, if $u=a s \in \mathscr{F}^{\prime \prime}$ and $v=b t \in \mathscr{S}^{\prime}$, where $s, t \in \mathscr{S}$, we have

$$
\lambda^{\prime 4}(u v)^{2}+\lambda^{\prime 2} Q^{\prime}\left(u, v^{\natural}\right) u v+\lambda^{\prime 2} Q^{\prime}(u) Q^{\prime}(v) 1=0 .
$$


But replacing $s$ by asa in (5.6) and comparing the resulting equation with (5.7), we get

$$
Q\left(\text { asa. } t^{\natural}\right) a s a t+Q(a s a) Q(t) 1=\rho\left(Q^{\prime}\left(u, v^{\natural}\right) u v+Q^{\prime}(u) Q^{\prime}(v) 1\right),
$$

where $\rho=\lambda^{2}\left(\lambda^{\prime}\right)^{-2}$. Since asat $\notin \ell 1$ for $(s, t)$ in a Zariski dense subset of $\mathscr{S} \times \mathscr{S}$, this forces $Q(a s a) Q(t)=\rho Q^{\prime}(a s) Q^{\prime}(a t)$ for $s . t \in \mathscr{S}$. Thus, $L_{a} \mid \mathcal{P}$ is a similarity of $Q$ onto $Q^{\prime}$.

Finally, the implication " $\Leftarrow$ " in (iii) follows from Proposition 5.2.

The implications " $\Rightarrow$ " in Theorem 5.4 remain to be proved. For that purpose, we assume for the rest of the section that $\beta: \mathscr{S} \mapsto \mathscr{S}^{\prime}$ is a similarity of $Q$ onto $Q^{\prime}$ with multiplier $\mu$.

LEMMA 5.8. There exists a unique isomorphism $\gamma: C^{+}(\mathscr{S}, Q) \mapsto C^{+}\left(\mathscr{S}^{\prime}, Q^{\prime}\right)$ such that

$$
\gamma\left(s_{C} t\right)=(1 / \mu)(\beta s)_{C}(\beta t)
$$

for $s, t \in \mathscr{P}$. (We use $x_{C} y$ for the products in both Clifford algebras.)

Proof. Define $\phi: \mathscr{S} \times \mathscr{P} \mapsto C^{+}\left(\mathscr{S}^{\prime}, Q^{\prime}\right)$ by $\phi(s, t)=(1 / \mu)(\beta s)_{C}(\beta t)$. Then,

$$
\begin{aligned}
\mu^{2} \phi(r, s)_{C} \phi(r, t) & =(\beta r)_{C}(\beta s)_{C}(\beta r)_{C}(\beta t) \\
& =-(\beta r)_{C}(\beta r)_{C}(\beta s)_{C}(\beta t)+Q^{\prime}(\beta r, \beta s)(\beta r)_{C}(\beta t) \\
& =-Q^{\prime}(\beta r)(\beta s)_{C}(\beta t)+Q^{\prime}(\beta r, \beta s)(\beta r)_{C}(\beta t) \\
& =\mu^{2}(-Q(r) \phi(s, t)+Q(r, s) \phi(r, t)) .
\end{aligned}
$$

Thus, the existence of a unique algebra homomorphism $\gamma$ satisfying (5.9) follows from Lemma 4.1. $\gamma$ is clearly surjective and hence injective.

LEMMA 5.10. The implications " $\Rightarrow$ " in Theorem 5.4(i) and (ii) are valid.

PROOF. If $m_{1}, m_{2} \leq 2$, then $\mathscr{H}(\mathscr{A},-) \cong \mathscr{H}^{\prime}\left(\mathscr{A}^{\prime},-\right)$ by Lemma 5.8 and Corollary 4.8. Suppose then that $\left\{m_{1}, m_{2}\right\}=\{4,1\},\{4,2\}$ or $\{4,4\}$. In that case, $(\mathscr{A},-)$ is associative and so $\Lambda=\mathscr{A}$. Thus, $\operatorname{End}_{\Lambda} \mathscr{A}=L_{\mathscr{A}} \cong \mathscr{A}$. Similarly, End $_{\Lambda^{\prime}} \mathscr{A}^{\prime} \cong \mathscr{A}^{\prime}$. Thus, by Lemma 5.8 and Corollary $4.8, \mathscr{A} \cong \mathscr{A}^{\prime}$ if $\left\{m_{1}, m_{2}\right\}=$ $\{4,1\}$ or $\{4,2\}$, and $\mathscr{A} \oplus \mathscr{A} \cong \mathscr{A}^{\prime} \oplus \mathscr{A}^{\prime}$ if $\left\{m_{1}, m_{2}\right\}=\{4,4\}$. Thus, in all three cases, $\mathscr{A} \cong \mathscr{A}^{\prime}$.

We are left with " $\Rightarrow$ " in Theorem 5.4(iii). So we assume for the rest of the section that $m_{1}=8$ and that $m_{2}=1,2,4$ or 8 . If $(\mathscr{A},-)\left(\operatorname{resp} .\left(\mathscr{A}^{\prime},-\right)\right)$ is a product algebra, we denote its factors by $\mathscr{C}_{1}, \mathscr{C}_{2}\left(\right.$ resp. $\left.\mathscr{C}_{1}^{\prime}, \mathscr{C}_{2}^{\prime}\right)$, where $\operatorname{dim}\left(\mathscr{C}_{1}\right)=8$ (resp. $\operatorname{dim}\left(\mathscr{C}_{1}^{\prime}\right)=8$ ). Then, by Proposition $2.2($ iii).

$$
\Lambda= \begin{cases}\mathscr{C}_{2} & \text { if } m_{2}=2 \text { or } 4, \\ \mathscr{C}_{1} & \text { if } m_{2}=1 \text { or } 8\end{cases}
$$

and a similar equation holds for $\Lambda^{\prime}$.

LEMMA 5.12. If $\left\{m_{1}, m_{2}\right\}=\{8,2\}$ or $\{8,4\}$, then $\mathscr{C}_{2} \cong \mathscr{C}_{2}^{\prime}$.

PROOF. Suppose first that $m_{2}=2$. Now $\operatorname{End}_{\Lambda} \mathscr{A} \cong \operatorname{End}_{\Lambda^{\prime}} \mathscr{A}^{\prime}$ by Lemma 5.8 and Corollary 4.8. If $\mathscr{C}_{2}$ is split, then $\operatorname{End}_{\Lambda} \mathscr{A}$ is not simple. This implies that $\operatorname{End}_{\Lambda^{\prime}} \mathscr{A}^{\prime}$ is not simple and so $\mathscr{C}_{2}^{\prime}$ is split. Thus, we can assume that $\Lambda$ and $\Lambda^{\prime}$ are fields. But then, since $\operatorname{End}_{\Lambda} \mathscr{A} \cong \operatorname{End}_{\Lambda^{\prime}} \mathscr{A}^{\prime}, \Lambda \cong \Lambda^{\prime}[\mathbf{1 7}$, Theorem 
4.7] and hence $\mathscr{C}_{2} \cong \mathscr{C}_{2}^{\prime}$. Suppose next that $m_{2}=4$. Then, as above, we get $\operatorname{End}_{\Lambda} \mathscr{A} \oplus \operatorname{End}_{\Lambda} \mathscr{A} \cong \operatorname{End}_{\Lambda^{\prime}} \mathscr{A}^{\prime} \oplus \operatorname{End}_{\Lambda^{\prime}} \mathscr{A}^{\prime}$. But $\Lambda$ and $\Lambda^{\prime}$ are central simple and hence so are End $\mathscr{E}_{\Lambda} \mathscr{A}$ and $\operatorname{End}_{\Lambda^{\prime}} \mathscr{A}^{\prime}\left[17\right.$, Theorem 4.7]. Thus, End $\mathscr{A}_{\Lambda} \cong \operatorname{End}_{\Lambda^{\prime}} \mathscr{A}^{\prime}$. But in the Brauer group, $[\Lambda]=\left[\operatorname{End}_{\Lambda} \mathscr{A}\right]^{-1}$ and $\left[\Lambda^{\prime}\right]=\left[\operatorname{End}_{\Lambda^{\prime}} \mathscr{A}^{\prime}\right]^{-1}$. Thus, $[\Lambda]=\left[\Lambda^{\prime}\right]$ and so $\Lambda \cong \Lambda^{\prime}$.

LEMMA 5.13. The implication " $\Rightarrow$ " in Theorem 5.4(iii) holds if $\left\{m_{1}, m_{2}\right\}=$ $\{8,2\}$ and the 2-dimensional factors are split.

PROOF. Now $\mathscr{P}\left(\mathscr{C}_{2},-\right)=h s_{2}$, where $s_{2}^{2}=1$. Hence, $n_{2}\left(s_{2}\right)=-1$. Thus, $Q$ is similar to the norm $n_{1}$ of $\mathscr{C}_{1}$. Similarly, $Q^{\prime}$ is similar to the norm $n_{1}^{\prime}$ of $\mathscr{C}_{1}^{\prime}$. Thus, there is a similarity $\rho$ of $n_{1}$ onto $n_{1}^{\prime}$. But then $\rho(1)$ is invertible and so $\phi=L_{\rho(1)}^{-1} \circ \rho$ is a similarity of $n_{1}$ onto $n_{1}^{\prime}$ such that $\phi(1)=1$. Thus $\phi$ is a norm equivalence and so $\mathscr{C}_{1} \cong \mathscr{C}_{1}^{\prime}\left[\mathbf{2 4}\right.$, Theorem 3.23]. Hence, $(\mathscr{A},-) \cong\left(\mathscr{A}^{\prime},-\right)$ and so $(\mathscr{A},-) \sim\left(\mathscr{A}^{\prime},-\right)$.

Thus, we may assume for the rest of the section that if $m_{2}=2$ then $\mathscr{C}_{2}$ and $\mathscr{C}_{2}^{\prime}$ are fields. Consequently, by (5.11), $\Lambda$ and $\Lambda^{\prime}$ are simple.

LEMMA 5.14. Replacing $\beta$ by another similarity if necessary, there exists a $\mathscr{R}$-linear bijection $\alpha: \mathscr{A} \mapsto \mathscr{A}^{\prime}$ such that

$$
\alpha L_{s} L_{t} \alpha^{-1}=(1 / \mu) L_{\beta s} L_{(\beta t)} \text { घ }
$$

for $s, t \in \mathscr{P}$.

ProOF. Now by Proposition 4.2, Theorem 4.5 and Lemma 5.8, we have homomorphisms $\gamma, \theta, \theta^{\prime}$ as indicated below, where $\gamma$ is an isomorphism and $\theta, \theta^{\prime}$ are surjections:

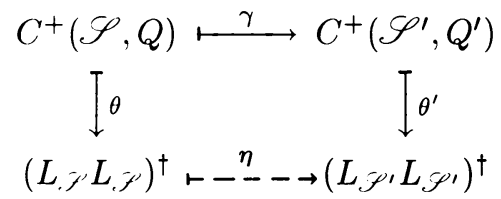

We wish to construct an isomorphism $\eta$ so that (5.16) commutes. If $m_{2}=1$ or 2 , then $\theta$ and $\theta^{\prime}$ are isomorphisms (by Theorem 4.5) and the existence of $\eta$ is clear. Suppose that $m_{2}=4$ or 8 . Then, the kernel of $\theta$ is one of the simple summands of $C^{+}(\mathscr{S}, Q)$ and a similar remark holds for $\theta^{\prime}$. But if $\rho: \mathscr{S} \mapsto \mathscr{S}$ is the $Q$-equivalence that sends one element in a $Q$-orthogonal basis to its negative and fixes the other elements in the basis, then the induced automorphism of $C^{+}(\mathscr{S}, Q)$ that sends $s_{C} t$ to $(\rho s)_{C}(\rho t)$ (Lemma 5.8) is not the identity map on the center of $C^{+}(\mathscr{S}, Q)$ (by $\left[\mathbf{1 7}\right.$, p. 237]), and hence it exchanges the simple summands of $C^{+}(\mathscr{S}, Q)$. Thus, replacing $\beta$ by $\beta \circ \rho$ if necessary, we may assume that $\gamma$ sends the kernel of $\theta$ onto the kernel of $\theta^{\prime}$, and so we have an isomorphism $\eta$ such that (5.16) commutes.

From the commutativity of (5.16), Proposition 4.2, and (5.9), it follows that

$$
\eta\left(L_{s} L_{t^{z}}\right)=(1 / \mu) L_{\beta s} L_{(\beta t)^{z}} .
$$

Also, by (4.6), $\eta$ is an isomorphism of $\operatorname{End}_{\Lambda} \mathscr{A}$ onto $\operatorname{End}_{\Lambda^{\prime}} \mathscr{A}^{\prime}$. Now if $m_{2}=1,2$, or 8 , our assumptions imply that $\Lambda$ and $\Lambda^{\prime}$ are fields, and so there exists a $k$-linear bijection $\alpha: \mathscr{A} \mapsto \mathscr{A}^{\prime}$ such that $\eta(A)=\alpha A \alpha^{-1}$ for $A \in \operatorname{End}_{\Lambda} \mathscr{A}[\mathbf{1 7}$, p. 206]. Thus, we may assume that $m_{2}=4$. But then since $\Lambda$ is central simple the natural 
mapping $\operatorname{End}_{\Lambda} \mathscr{A} \otimes R_{\Lambda} \mapsto \operatorname{End}_{\not} \mathscr{A}$ is an algebra isomorphism [17, Theorem 4.7], and a similar remark holds for $\left(\mathscr{A}^{\prime},-\right)$. But by Lemma $5.12, \Lambda \cong \Lambda^{\prime}$ and hence $R_{\Lambda} \cong R_{\Lambda^{\prime}}$. Thus, $\eta$ extends to an isomorphism $\zeta$ of End $\mathscr{A}_{\mathscr{A}}$ onto End $\mathscr{A}^{\prime}$. But then again there exists a $\ell$-linear bijection $\alpha: \mathscr{A} \mapsto \mathscr{A}^{\prime}$ such that $\zeta(A)=\alpha A \alpha^{-1}$ for $A \in \operatorname{End}_{k} \mathscr{A}$.

The remainder of the proof of Theorem 5.4 consists of showing that $\alpha$ is an isotopy of $(\mathscr{A},-)$ onto $\left(\mathscr{A}^{\prime},-\right)$.

Now $(\mathscr{A},-)$ is simple and hence $\mathscr{K}=\mathscr{K}(\mathscr{A},-)$ is simple. Also,

$$
\mathscr{K}=\bigoplus \sum_{i \in \mathbf{Z}} \mathscr{K}_{i}=\mathscr{K}_{-2} \oplus \mathscr{K}_{-1} \oplus \mathscr{K}_{0} \oplus \mathscr{K}_{1} \oplus \mathscr{K}_{2}
$$

and $\mathscr{K}_{i}$ is the $i$-eigenspace for $\operatorname{ad}\left(V_{1,1}\right)$. Thus, $\mathscr{K}_{i}$ and $\mathscr{K}_{j}$ are orthogonal with respect to the Killing form if $i \neq-j$. Also, $\left[\mathscr{K}_{0}, \mathscr{K}_{i}\right]=\mathscr{K}_{i}$ for $i \neq 0,|i| \leq 2$. Finally, $\mathscr{K}_{-1}, \mathscr{K}_{0}$, and $\mathscr{K}_{1}$ generate the Lie algebra $\mathscr{K}$. Similar remarks hold for $\mathscr{K}^{\prime}=\mathscr{K}\left(\mathscr{A}^{\prime},-\right)$. But these are precisely the conditions required to invoke a lemma of Seligman on extending isomorphisms [26, Lemma 4.2] that implies the following.

LemMA 5.17. Suppose $\omega_{i}: \mathscr{K}_{i} \mapsto \mathscr{K}_{i}^{\prime}$ are $/$-linear bijections, $i=0,1,2$, such that

$$
\omega_{i}\left[X_{0}, Y_{i}\right]=\left[\omega_{0} X_{0}, \omega_{i} Y_{i}\right]
$$

for $X_{0} \in \mathscr{K}_{0}, Y_{i} \in \mathscr{K}_{i}, i=0,1,2$. Then, there exists a unique Lie algebra isomorphism $\omega: \mathscr{K} \mapsto \mathscr{K}^{\prime}$ such that $\left.\omega\right|_{\mathscr{K}_{0}}=\omega_{0},\left.\omega\right|_{\mathscr{K}_{1}}=\omega_{1}$, and $\omega\left(\mathscr{K}_{-1}\right)=\mathscr{K}_{-1}^{\prime}$.

LEMMA 5.19. The implication " $\Rightarrow$ " in Theorem $5.4(\mathrm{iii})$ is valid.

ProOF. By (5.15) and (4.6), $\alpha \operatorname{End}_{\Lambda} \mathscr{A} \alpha^{-1}=\operatorname{End}_{\Lambda^{\prime}} \mathscr{A}^{\prime}$. But since $\Lambda$ is simple, $R_{\Lambda}$ is the centralizer of $\operatorname{End}_{\Lambda} \mathscr{A}$ in $\operatorname{End}_{\not{A}} \mathscr{A}$ [17, Theorem 4.10]. Moreover, a similar remark holds for $\left(\mathscr{A}^{\prime},-\right)$. Hence, $\alpha R_{\Lambda} \alpha^{-1}=R_{\Lambda^{\prime}}$. But by $(5.11),(\Lambda,-)$ and $\left(\Lambda^{\prime},-\right)$ are (associative) composition algebras. Hence, any isomorphism of $\Lambda$ onto $\Lambda^{\prime}$ preserves the involutions. Thus, $\alpha R_{\mathcal{F}^{\prime} \cap \Lambda} \alpha^{-1}=R_{\text {, }{ }^{\prime} \cap \Lambda^{\prime}}$. Consequently, by Theorem 4.4, $\alpha \cdot \mathscr{K}_{0} \alpha^{-1}=\mathscr{K}_{0}^{\prime}$.

Define $\omega: \mathscr{K}_{i} \mapsto \mathscr{K}_{i}{ }^{\prime}, i=1,2$, as follows.

$$
\omega_{0}(A)=\alpha A \alpha^{-1}, \quad \omega_{1}(x, 0)=(\alpha x, 0), \quad \omega_{2}(0, s)=(0, \beta s)
$$

for $A \in \mathscr{K}_{0}, x \in \mathscr{A}, s \in \mathscr{P}$. We wish to apply Lemma 5.17 and so we must verify (5.18) for $i=0,1,2$. The case $i=0$ is clear. Next if $X_{0}=A \in \mathscr{K}_{0}$ and $Y_{1}=(y, 0) \in \mathscr{K}_{1}$, then

$$
\omega_{1}\left[X_{0}, Y_{1}\right]=\omega_{1}(A y, 0)=(\alpha A y, 0)=\left(\alpha A \alpha^{-1} \alpha y, 0\right)=\left[\omega_{0} X_{0}, \omega_{1} Y_{1}\right] .
$$

Finally, we must show that $\omega_{2}[A,(0, r)]=\left[\omega_{0} A, \omega_{2}(0, r)\right]$ or equivalently that

$$
\beta\left(A^{\delta} r\right)=\left(\alpha A \alpha^{-1}\right)^{\delta} \beta r
$$


for $A \in \mathscr{K}_{0}$ and $r \in \mathscr{S}$. But $\left.R_{\mathscr{Y} \cap \Lambda}^{\delta}\right|_{\mathscr{\gamma}}=\{0\}$ and $R_{\mathscr{P}^{\prime} \cap \Lambda^{\prime}}^{\delta} \mid \mathscr{P}^{\prime}=\{0\}$. Thus, by Theorem 4.4, it suffices to prove (5.20) for $A=L_{s} L_{t^{\natural}}$, where $s, t \in \mathscr{S}$. But then

$$
\begin{aligned}
\beta\left(A^{\delta} r\right) & =\beta\left(\left(L_{s} L_{t^{\sharp}}\right)^{\delta} r\right)=\beta\left(s\left(t^{\natural} r\right)+r\left(t^{\natural} s\right)\right) \quad(\text { by }(1.1)) \\
& =\beta(Q(s, r) t-Q(s, t) r-Q(r, t) s) \quad(\text { by }(3.7)) \\
& =(1 / \mu)(Q(\beta s, \beta r) \beta t-Q(\beta s, \beta t) \beta r-Q(\beta r, \beta t) \beta s) \\
& =(1 / \mu)\left((\beta s)\left((\beta t)^{\natural}(\beta r)\right)+(\beta r)\left((\beta t)^{\natural}(\beta s)\right)\right) \quad \text { by }(3.7 \\
& =(1 / \mu)\left(L_{\beta s} L_{(\beta t)^{\natural}}\right)^{\delta} \beta r \quad(\text { by }(1.1)) \\
& =\left(\omega_{0} A\right)^{\delta} \beta r \quad(\text { by }(5.15)) .
\end{aligned}
$$

This proves (5.20) and hence (5.18).

By Lemma 5.17, there exists an isomorphism $\omega: \mathscr{K} \mapsto \mathscr{K}^{\prime}$ such that $\left.\omega\right|_{\mathscr{K}_{0}}=\omega_{0}$, $\omega \mid \mathscr{K}_{1}=\omega_{1}$, and $\omega\left(\mathscr{K}_{-1}\right)=\mathscr{K}_{-1}^{\prime}$. Define $\hat{\alpha}: \mathscr{A} \mapsto \mathscr{A}^{\prime}$ by $(\hat{\alpha} x, 0)^{\sim}=\omega(x, 0)^{\sim}$. Then,

$$
\begin{aligned}
(\alpha\{x, y, z\}, 0) & =\omega\left[\left[(x, 0),(y, 0)^{\sim}\right],(z, 0)\right] \\
& =\left[\left[(\alpha x, 0),(\hat{\alpha} y, 0)^{\sim}\right],(\alpha z, 0)\right]=(\{\alpha x, \hat{\alpha} y, \alpha z\}, 0)
\end{aligned}
$$

for $x, y, z \in \mathscr{A}$ and so $\alpha$ is an isotopy.

This completes the proof of Theorem 5.4.

REMARK. Among the lemmas obtained in the proof of Theorem 5.4, Lemma 5.12 is of independent interest as it states that the lower dimensional factor of an $(8,2)$ - or (8,4)-product algebra is an isotopy invariant. This lemma also follows from Remark (ii) in $\S 2$, Theorem 1.3, and the fact that a composition algebra of dimension 2 or 4 used (along with an exceptional central simple Jordan algebra) in the second Tits' Lie algebra construction is an isomorphism invariant of the resulting Lie algebra (see $[\mathbf{1 0}, \S 3$ and $\mathbf{3 0}$, p. 215]).

6. Central simple Lie algebras of index $F_{4,1}^{21},{ }^{2} E_{6,1}^{29}, E_{7,1}^{48}$ and $E_{8,1}^{91}$. In this section, we apply the results obtained so far to give a description of the isomorphism classes of central simple Lie algebras with index

$$
F_{4,1}^{21}, \quad{ }^{2} E_{6,1}^{29}, \quad E_{7,1}^{48}, \quad \text { or } \quad E_{8,1}^{91} \text {. }
$$

Recall that the type of a semisimple Lie algebra $\mathscr{L}$ is the type of $\mathscr{L}_{\Omega}$, where $\Omega$ is the algebraic closure of $h$. If $X_{n}$ is one of the exceptional simple types $\left(D_{4}, G_{2}, F_{4}, E_{6}, E_{7}\right.$ or $\left.E_{8}\right)$, a Lie algebra $\mathscr{L}$ is said to have index ${ }^{g} X_{n, r}^{t}$ if $\mathscr{L}$ is central simple of type $X_{n}, n$ is the rank of $\mathscr{L}_{\Omega}, r$ is the relative rank of $\mathscr{L}, r+t$ is the dimension of the centralizer of a maximal split toral subalgebra of $\mathscr{L}$, and $g$ is the order of the quotient of the Galois group $\operatorname{Gal}(\Omega / k)$ that operates effectively on the Dynkin diagram of $\mathscr{L}_{\Omega}\left[29\right.$, p. 54]. If $X_{n}=G_{2}, F_{4}, E_{7}$ or $E_{8}, g$ must be 1 and so $g$ is omitted from the notation.

The following result, which is in part a summary of some of our earlier results, shows that the classification of central simple Lie algebras with index (6.1) is equivalent to the classification up to similarity of certain Albert forms.

THEOREM 6.2. Suppose $m=1,2,4$ or 8 and $I=F_{4,1}^{21},{ }^{2} E_{6,1}^{29}, E_{7,1}^{48}$ or $E_{8,1}^{91}$ accordingly. Suppose $\mathscr{L}$ is a Lie algebra over $\mathscr{k}$. Then, the following are equivalent:

(a) $\mathscr{L}$ is central simple of index $I$. 
(b) $\mathscr{L} \cong \mathscr{K}(\mathscr{A},-)$, where $(\mathscr{A},-)$ is a division algebra form of an $(8, m)$-product algebra.

(c) $\mathscr{L} \cong \mathscr{K}(\mathscr{A},-)$, where $(\mathscr{A},-)$ is a form of an $(8, m)$-product algebra such that the Albert form of $(\mathscr{A},-)$ is anisotropic and, if $m=2$, the 2-dimensional factor of $(\mathscr{A},-)$ is a field.

Suppose further that $(\mathscr{A},-)$ and $\left(\mathscr{A}^{\prime},-\right)$ are as in $(\mathrm{b})$ or $(\mathrm{c})$ with Albert forms $Q$ and $Q^{\prime}$ respectively. Then the following are equivalent:

$(\alpha) \mathscr{K}(\mathscr{A},-) \cong \mathscr{K}\left(\mathscr{A}^{\prime},-\right)$.

( $\beta)(\mathscr{A},-) \sim\left(\mathscr{A}^{\prime},-\right)$.

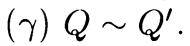

Moreover, if $m=1$ or 2 , then $(\alpha),(\beta)$, and $(\gamma)$ are equivalent to each of the following:

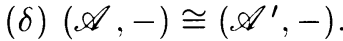

$(\varepsilon)$ The factors of $(\mathscr{A},-)$ are isomorphic to the factors of $\left(\mathscr{A}^{\prime},-\right)$.

PROOF. The equivalence of (b) and (c) follows from Theorem 3.14 and Proposition 2.2(iv). The equivalence of $(\alpha),(\beta)$ and $(\gamma)$ follows from Theorems 1.3 and 5.4 (iii). The equivalence of $(\beta),(\delta)$ and $(\varepsilon)$ in the case $m \leq 2$ follows from Theorem 5.1. Thus, we need only prove the equivalence of (a) and (b).

"(b) $\Rightarrow($ a)" Suppose that $(\mathscr{A},-)$ is a division algebra form of an $(8, m)$-product algebra. By Proposition $2.2(\mathrm{v}),(\mathscr{A},-)$ is a central division algebra. Thus, by Theorem $1.2, \mathscr{K}(\mathscr{A},-)$ is central simple of relative rank 1 . But $\ell V_{1,1}$ is a split toral subalgebra of $\mathscr{K}(\mathscr{A},-)$ and hence it is a maximal split toral subalgebra. But the centralizer of $\mathscr{R} V_{1,1}$ in $\mathscr{K}(\mathscr{A},-)$ is $\mathscr{K}_{0}=\operatorname{Instrl}(\mathscr{A},-)$ and

$$
\begin{aligned}
\operatorname{dim}\left(\mathscr{K}_{0}\right) & =\operatorname{dim}(\mathscr{P} \cap \Lambda)+\operatorname{dim}(L, L, \quad \text { (by Theorem 4.4) } \\
& =\operatorname{dim}(\mathcal{P} \cap \Lambda)+1+\operatorname{dim}(\circ(\mathscr{P}, Q)) \quad \text { (by Proposition 4.3) } \\
& =\operatorname{dim}(\mathscr{P} \cap \Lambda)+1+\left(\begin{array}{l}
d \\
2
\end{array}\right) \quad(\text { where } d=\operatorname{dim}(\mathscr{P})) \\
& =22,30,49 \text { or } 92 \text { according as } m=1,2,4 \text { or } 8 .
\end{aligned}
$$

Thus, since there are no central simple Lie algebras with index ${ }^{1} E_{6,1}^{29}$ [29, p. 58], it remains only to show that $\mathscr{K}(\mathscr{A},-)_{\Omega}$ has type $F_{4}, E_{6}, E_{7}$ or $E_{8}$ according as $m=1,2,4$ or 8 . But

$$
\begin{aligned}
\operatorname{dim}(\mathscr{K}(\mathscr{A},-)) & =2 \operatorname{dim}(\mathscr{P})+2 \operatorname{dim}(\mathscr{A})+\operatorname{dim}(\operatorname{Instrl}(\mathscr{A},-)) \\
& =52,78,133 \text { or } 248 \text { according as } m=1,2,4 \text { or } 8 .
\end{aligned}
$$

Since $F_{4}, E_{7}$ or $E_{8}$ is the unique type of a simple Lie algebra over $\Omega$ of dimension 52 , 133 or 248 respectively, we may assume that $m=2$. Hence, $\operatorname{dim}(\mathscr{K}(\mathscr{A},-))=78$ and so $\mathscr{K}(\mathscr{A},-)$ has type $B_{6}, C_{6}$ or $E_{6}$. Also $\left[\mathscr{K}_{0}, \mathscr{K}_{0}\right] \cong o(\mathscr{P}, Q)$ is central simple of type $D_{4}$. But in any central simple algebra of type $B_{6}$ or $C_{6}$ and relative rank 1 , the derived algebra of the centralizer of a maximal split toral subalgebra is semisimple of type $B_{5}, A_{1} \oplus C_{4}$ or $A_{3} \oplus B_{2}$ (by the last paragraph of $[\mathbf{2 9}, \S 2.3]$ and the tables on pp. 5556 of $[\mathbf{2 9}])$. Thus, $\mathscr{K}(\mathscr{A},-)$ has type $E_{6}$.

"(a) $\Rightarrow($ b)" Suppose $\mathscr{L}$ is central simple of index $I$. Thus, $\mathscr{L}$ has relative rank 1 and so, by Theorem 1.2 , we may assume that $\mathscr{L}=\mathscr{K}(\mathscr{A},-)$ for some central structurable division algebra $(\mathscr{A},-)$. Then $\mathscr{T}=\mathscr{R} V_{1,1}$ is a maximal split toral subalgebra of $\mathscr{L}$. Let $\mathscr{M}$ be a ('artan subalgebra of $\mathscr{L}$ containing $\mathscr{T}$, and $\Sigma$ be the 
roots of $\mathscr{M}_{\Omega}$ in $\mathscr{L}_{\Omega}$. We may order $\Sigma$ so that the simple system $\Delta=\left\{\alpha_{1}, \ldots, \alpha_{n}\right\}$ of roots satisfies $\alpha_{i}\left(V_{1,1}\right)=0$ or 1 for all $i$. The $\alpha_{i}$ such that $\alpha_{i}\left(V_{1,1}\right)=1$ are the simple roots contained in the circled orbit on the diagrams in [29, Table II]. Examining the diagrams for the given indices, we see that there is in each case more than one root $\alpha=\sum n_{i} \alpha_{i}$ such that the sum over the circled orbit of the $n_{i}$ 's is 2. Thus, $\operatorname{dim}(\mathscr{P})=\operatorname{dim}\left(\mathscr{K}_{2}\right)>1$. Consequently, $\mathscr{K}(\mathscr{A},-)$ is central simple of type $F_{4}, E_{6}, E_{7}$ or $E_{8}$ and $\operatorname{dim}(\mathscr{S})>1$. Hence, according to the case-by-case examination of the possibilities for $\mathscr{K}(\mathscr{A},-)$ given in $[\mathbf{5}, \S 8]$, it follows that $(\mathscr{A},-)$ is a form of an $(8, m)$-product algebra.

REMARKS. (i) In [26, pp. 242-258] and [27, Chapters 5 and 6], Seligman has given constructions that yield all Lie algebras of index (6.1) starting from a nondegenerate quadratic form $q$ defined on a vector space $\mathscr{V}$ and an irreducible module for the Clifford algebra $C(\mathscr{V}, q)$. The quadratic form $q$ is assumed to not represent $1, \mathscr{V}$ is assumed to have dimension $6,7,9$ or 13 respectively, and the Clifford algebra $C(\mathscr{V}, q)$ is assumed to have a specified structure. For example, in the case $\operatorname{dim}(\mathscr{V})=7, C(\mathscr{V}, q)$ is assumed to be a full matrix algebra over a quadratic extension of $\mathscr{l}$. Now if $(\mathscr{A},-)$ is a division algebra form of an $(8, m)$-product algebra, then we may fix an element $s_{0} \neq 0 \in \mathscr{S}$ and put $\mathscr{V}=\left(\ell s_{0}\right)^{\perp}$ (with respect to the Albert form $Q$ ) and $q=-\left(1 / Q\left(s_{0}\right)\right) Q \mid \%$. Then, $q$ does not represent 1 (since $Q$ is anisotropic), and $\operatorname{dim}(\mathscr{V})=6,7,9$, or 13 according as $m=1,2,4$ or 8 . Moreover, we have $C(\mathscr{V}, q) \cong C^{+}(\mathscr{S}, Q)[\mathbf{1 7}$, Theorem 4.13]. Thus, in view of Corollary 4.8 , we have certain restrictions on the structure of $C(\mathscr{V}, q)$. Those restrictions are precisely the structural restrictions assumed by Seligman in his constructions. The Lie algebra constructed by Seligman from $q$ is undoubtably isomorphic to $\mathscr{K}(\mathscr{A},-)$, although we have not checked that assertion.

(ii) Any Lie algebra of type $F_{4}$ is isomorphic to the derivation algebra of an exceptional central simple Jordan algebra, and two such Lie algebras are isomorphic if and only if the corresponding Jordan algebras are isomorphic [31, Theorems 4 and 2]. Theorem 6.2 in the case $m=1$ (see $[\mathbf{2 9}$, p. 61]) can also be easily proved using this fact, Remark (ii) of $\S 2$, and standard results about exceptional central simple Jordan algebras.

We conclude this section by applying Theorem 6.2 to obtain new proofs of some known descriptions of Lie algebras of index (6.1) over real closed fields, local fields, and number fields. We require some notation.

First of all, if $\delta_{1}, \ldots, \delta_{r} \in R$, we denote by $\left\langle\delta_{1}, \ldots, \delta_{r}\right\rangle$ the quadratic form $q\left(x_{1}, \ldots, x_{r}\right)=\delta_{1} x_{1}^{2}+\cdots+\delta_{r} x_{r}^{2}$ defined on $k^{r}$. We use the symbol $\cong$ for equivalence of quadratic forms.

Next if $\alpha_{1}, \ldots, \alpha_{l} \neq 0 \in h$, where $0 \leq l \leq 3$, we denote by

$$
\left(\frac{\alpha_{1}, \ldots, \alpha_{l}}{k}\right)
$$

the composition algebra of dimension $2^{l}$ obtained by $l$ applications of the CayleyDickson process starting from $k$ and using the scalars $\alpha_{1}, \ldots, \alpha_{l}$ [24, pp. 45-46]. If the base field is understood from the context, we also use the rotation $\left(\alpha_{1}, \ldots, \alpha_{l}\right)$ for this algebra. Any composition algebra arises in this way [14, Theorem 1]. If $l=1,2$ or 3 , the pure norm of $\left(\alpha_{1}, \ldots, \alpha_{l}\right)$ is equivalent respectively to

$$
\begin{gathered}
\left\langle-\alpha_{1}\right\rangle, \quad\left\langle-\alpha_{1},-\alpha_{2}, \alpha_{1} \alpha_{2}\right\rangle, \quad \text { or } \\
\left\langle-\alpha_{1},-\alpha_{2},-\alpha_{3}, \alpha_{1} \alpha_{2}, \alpha_{1} \alpha_{3}, \alpha_{2} \alpha_{3},-\alpha_{1} \alpha_{2} \alpha_{3}\right\rangle .
\end{gathered}
$$


Suppose now that $h$ is real closed. Then, the composition division algebras of dimension $>1$ are $(-1),(-1,-1)$, and $(-1,-1,-1)$. By $(6.3)$, the pure norm of any of these algebras represents 1 , and so the Albert form of the tensor product of any two of them is isotropic. Also the unique quadratic extension $k(\sqrt{-1})$ of $h$ is algebraically closed [21, Theorem VII.2.5], and so the Albert form of any twisted (8,8)-product algebra is isotropic. Thus, there is one $(8,1)$-product division algebra $((-1,-1,-1),-)$ and no division algebra forms of $(8, m)$-product algebras for $m>1$. Correspondingly, there is one Lie algebra with index $F_{4,1}^{21}$ over $h$ and none with index ${ }^{2} E_{6,1}^{29}, E_{7,1}^{48}$ or $E_{8,1}^{91}$.

Suppose next that $h$ is a local field, by which we mean a field with complete discrete valuation and finite residue class field. Then, there are no anisotropic quadratic forms of dimension $>4[\mathbf{1 7}$, Proposition 9.11] and hence there are no Lie algebras with index (6.1) over $h$.

Suppose finally that $\ell$ is an algebraic number field. Let $\sigma_{1}, \ldots, \sigma_{t}$ be the distinct monomorphisms of $\mathscr{k}$ into the field $\mathbf{R}$ of real numbers. (We may have $t=0$.) Then Albert and Jacobson [2, pp. 414-415] have shown that any octonion algebra over $k$ is isomorphic to $(-1,-1, \gamma)$ for some $\gamma \neq 0 \in k$. They also showed that two such algebras $(-1,-1, \gamma)$ and $\left(-1,-1, \gamma^{\prime}\right)$ are isomorphic if and only if $\sigma_{i}\left(\gamma \gamma^{\prime}\right)>0$ for all $i$, and that $(-1,-1, \gamma)$ is a division algebra if and only if $\sigma_{i}(\gamma)<0$ for some $i$. Hence, there are (up to isomorphism) $2^{t}-1$ octonion division algebras over $k$. Correspondingly, there are (up to isomorphism) $2^{t}-1$ Lie algebras with index $F_{4,1}^{21}$. Next, it follows from the above description of the octonion algebras over $k$ and from (6.3) that the pure norm of any octonion algebra over $k$ (or over a quadratic extension of $\ell$ ) represents 1 . Consequently, the Albert form of any (twisted or untwisted) form of an $(8,8)$-product algebra over $\mathscr{Z}$ is isotropic. Thus, there are no Lie algebras of index $E_{8,1}^{91}$ over $\mathscr{l}$. Next suppose that $(\mathscr{A},-)=\left(\mathscr{C}_{1},-\right) \otimes\left(\mathscr{C}_{2},-\right)$ is an $(8,4)$-product algebra. Now $\mathscr{C}_{1}$ contains a quaternion subalgebra $\mathscr{B}_{1}$. But then there exists $\alpha, \beta, \gamma \in \ell$ such that $\mathscr{B}_{1}=(\alpha, \beta)$ and $\mathscr{C}_{2}=(\alpha, \gamma)$ [21, p. 171]. Consequently, the Albert form of $\left(\mathscr{B}_{1},-\right) \otimes\left(\mathscr{C}_{2},-\right)$ is isotropic. Thus, the Albert form of $(\mathscr{A},-)$ is isotropic. Thus, there are no Lie algebras of index $E_{7,1}^{48}$ over $h$. The final index ${ }^{2} E_{6,1}^{29}$ is covered by the following.

PROPOSITION 6.4. Suppose $\mathscr{l}$ is a number field and $\sigma_{1}, \ldots, \sigma_{t}$ are the distinct monomorphisms of $\mathscr{h}$ into $\mathbf{R}$. Let $\not{ }^{\text {pos }}=\left\{\alpha \in \ell \mid \sigma_{i}(\alpha)>0\right.$ for all $\left.i\right\}$, $\mathscr{K}^{\times}=\{\alpha \in \mathscr{R} \mid \alpha \neq 0\}$, and $G=\left(\mathscr{K}^{\times} / \mathscr{K}^{\mathrm{pos}}\right) \times\left(\mathscr{K}^{\times} / \mathscr{K}^{\times 2}\right)$. Then, the mapping $\left(\gamma / \ell^{\text {pos }}, \delta \ell^{\times 2}\right) \mapsto((-1,-1, \gamma),-) \otimes((\delta),-)$ is a bijection of the group $G$ onto a set of representatives of the distinct isotopy classes of $(8,2)$-product algebras. Moreover, the pair $\left(\gamma / h^{\text {pos }}, \delta /{ }^{\times 2}\right)$ is mapped onto a division algebra if and only if

$$
\delta \notin \ell^{\times 2} \text { and, for some } i, \sigma_{i}(\gamma)<0 \text { and } \sigma_{i}(\delta)>0 \text {. }
$$

PROOF. The first statement follows from Theorem 5.1 and the results of Albert and Jacobson mentioned above. For the last statement, put

$$
(\mathscr{A},-)=((-1,-1, \gamma),-) \otimes((\delta),-) .
$$

The Albert form $Q$ of $(\mathscr{A},-)$ is (by $(6.3))$ equivalent to $\langle 1,1,1,-\gamma,-\gamma,-\gamma,-\gamma, \delta\rangle$. Thus, by [21. Corollary 3.5], $Q$ is anisotropic if and only if $\sigma_{i}(\gamma)<0$ and $\sigma_{i}(\delta)>0$ for some $i$. Since $(\delta)$ is a field if and only if $\delta \notin \ell \times 2$, the statement follows from Theorem 6.2. 
COROLLARY 6.6. If $t>0$, there exist infinitely many isomorphism classes of Lie algebras of index ${ }^{2} E_{6,1}^{29}$ over the number field $k$.

PROOF. It suffices to show that there exist infinitely many pairwise nonisotopic $(8,2)$-product division algebras of the form $((-1,-1,-1),-) \otimes((\delta),-)$. Thus, by the proposition, it suffices to show there exist infinitely many nontrivial cosets $\delta /{ }^{\times 2}$ in $k^{\times} / \ell^{\times 2}$ such that $\sigma_{i}(\delta)>0$ for some $i$. In fact there exist infinitely many with $\sigma_{i}(\delta)>0$ for all $i$. This follows from the fact that $\left(k^{\text {pos }}: k^{\times 2}\right)$ is infinite, which in turn follows from the fact that $\left(\mathscr{K}^{\times}: \mathscr{K}^{\times 2}\right)$ is infinite [21, p. 216] and the fact that $\left(\mathscr{Z}^{\times}: \mathscr{K}^{\text {pos }}\right)$ is finite.

REMARKS. (i) If $\mathscr{l}$ is a number field, it follows from Proposition 6.4 and Theorem 6.2 that the mapping

$$
\left(\gamma \mathscr{k}^{\text {pos }}, \delta \mathscr{R}^{\times 2}\right) \mapsto \mathscr{K}(((-1,-1, \gamma),-) \otimes((\delta),-))
$$

is a bijection of the set of elements of $G$ satisfying (6.5) onto the set of isomorphism classes of Lie algebras of index ${ }^{2} E_{6,1}^{29}$. The fact that this mapping is well defined and one-to-one can also be deduced from a more general isomorphism criterion due to Brown $[9 ; 11$, Theorem 7.2$]$ for certain Lie algebras of type $E_{6}$ over a number field. We also note that Ferrar has shown that all Lie algebras of type $E_{6}$ over a number field can be constructed using Tits' second construction [11, Theorem 6.4].

(ii) For all possible indices $I$, the existence or nonexistence of Lie algebras of index $I$ over $\mathbf{R}, p$-adic fields, or number fields has been tabulated in Table II of [29]. The above discussion confirms the entries in that table corresponding to the indices (6.1).

7. The base field $k_{n}=\mathbf{R}\left(\left(T_{1}, \ldots, T_{n}\right)\right)$. It is interesting to note that Lie algebras of index $E_{7,1}^{48}$ and $E_{8,1}^{91}$ do not exist over any of the special fields considered so far. However, Lie algebras of index $E_{7,1}^{48}$ and $E_{8,1}^{91}$ are known to exist over $\mathbf{Q}\left(T_{1}\right)$ and $\mathbf{Q}\left(T_{1}, T_{2}\right)$ respectively, where $T_{1}$ and $T_{2}$ are indeterminants and $\mathbf{Q}$ is the field of rationals $[\mathbf{2 7}, \S 6.3]$. We wish now to consider a base field over which Lie algebras of all the indices (6.1) exist and can be classified, namely the field $\mathbf{R}\left(\left(T_{1}, T_{2}, T_{3}\right)\right)$ of iterated Laurent series in 3 variables over the real field $\mathbf{R}$. Our methods, at least in principle, are applicable more generally to algebras over

$$
\ell_{n}=\mathbf{R}\left(\left(T_{1}, \ldots, T_{n}\right)\right)=\mathbf{R}\left(\left(T_{1}\right)\right) \cdots\left(\left(T_{n}\right)\right),
$$

where $n \geq 0$. We begin by establishing the nonexistence of twisted $(8,8)$-product division algebras over $\ell_{n}$.

If $E / F$ is a quadratic extension, we use the notation $H(E / F)$ to denote the subgroup of $E^{\times}$defined by

$$
H(E / F)=E^{\times 2}\left\{\beta \in E^{\times} \mid \beta^{2} \in F^{\times}\right\} .
$$

LEMMA 7.1. Suppose that $H(K / \mathscr{k})=K^{\times}$for every quadratic extension $K$ of $k$. Then, there does not exist a twisted $(4,4)$ or $(8,8)$-product division algebra over $k$.

PROOF. Since any octonion algebra contains a quaternion algebra, it suffices to prove the nonexistence of twisted $(4,4)$-product division algebras over $\not{k}$. Suppose then that $(\mathscr{A},-)=\operatorname{cor}_{K / \mathscr{k}}(\mathscr{C},-)$, where $(\mathscr{C},-)$ is a quaternion algebra over a quadratic extension $K=\ell(\sqrt{\delta})$ of $h$. Now $\mathscr{C}=\left(\frac{\alpha, \beta}{K}\right)$ for some $\alpha, \beta \in K^{\times}$. But 
then since $H(K / \ell)=K^{\times}$, we may assume that $\alpha^{2} \in k^{\times}$and $\beta^{2} \in k^{\times}$. Thus, $\alpha, \beta \in \ell^{\times} \cup h^{\times} \sqrt{\delta}$. Hence, either $-\alpha,-\beta$ or $\alpha \beta \in h^{\times}$. But $-\alpha,-\beta$ and $\alpha \beta$ are represented by the pure norm of $\mathscr{C}$. Hence, the pure norm of $\mathscr{C}$ represents an element of $\ell^{\times}$and so the Albert form of $(\mathscr{A},-)$ is isotropic. The lemma now follows from Corollary 3.15 .

LEMMA 7.2. Suppose $\ell$ has a complete discrete valuation with residue class field $F$ of characteristic $\neq 2$. If $H(E / F)=E^{\times}$for all quadratic extensions $E$ of $F$, then $H(K / \ell)=K^{\times}$for all quadratic extensions $K$ of $k$.

PROOF. Suppose $K$ is a quadratic extension of $k$. The valuation of $k$ extends uniquely to a complete discrete valuation on $K[\mathbf{1 7}$, Proposition 9.3$]$. We use the notation $R_{k}, R_{\not k}^{\times}$, and $-: R_{\not k} \mapsto \bar{k}=F$ for respectively the valuation $\operatorname{ring}$ of $k$, the group of units of $R_{k}$, and the canonial map of $R_{k}$ onto the residue class field $\bar{k}$. (We suspend for this proof our use of the notation - for an involution.) We use similar notation $R_{K}, R_{K}^{\times}$, and $-: R_{K} \mapsto \bar{K}$ for $K$.

We first claim that there exists a prime $\pi$ of $R_{K}$ such that $\pi^{2} \in k^{\times}$. Indeed if $K / \mathscr{k}$ is unramified this is clear since a prime of $R_{k}$ is a prime of $R_{K}$. On the other hand, if $K / \mathscr{R}$ is not unramified, then $K / \ell$ is totally ramified (since $[K: \ell]=2$ ) and so any prime $\pi_{K}$ of $R_{K}$ is a root of an Eisenstein polynomial $f(x)=x^{2}+a x+b$ over $R_{k}\left[\mathbf{2 2}\right.$, p. 46]. In that case, we may take $\pi=\pi_{K}+a / 2$.

Now $K^{\times}$is generated by $\pi$ and $R_{K}^{\times}$. Since $\pi^{2} \in \ell^{\times}$, it suffices to show that $R_{K}^{\times} \subseteq H(K / /)$. Let $\alpha \in R_{K}^{\times}$. Then, $\bar{\alpha} \in \bar{K}^{\times}$. Hence, $\bar{\alpha}=\bar{\beta}^{2} \bar{\gamma}$ for some $\beta, \gamma \in R_{K}^{\times}$ such that $\bar{\gamma}^{2} \in \bar{h}^{\times}$. (This is trivial if $\bar{K}=\bar{h}$ and it follows by hypothesis otherwise.) Then, by Hensel's lemma, there exists $\delta \in R_{K}^{\times}$such that $\delta^{2} \in \ell^{\times}$and $\bar{\delta}=\bar{\gamma}$. Thus, $\bar{\alpha}=\bar{\beta}^{2} \bar{\delta}$, where $\delta^{2} \in \ell^{\times}$. Replacing $\alpha$ by $\alpha \delta^{-1}$, we may assume that $\bar{\alpha}=\bar{\beta}^{2}$, where $\beta \in R_{K}^{\times}$. But then again by Hensel's lemma, $\alpha \in R_{K}^{\times 2} \subseteq H(K / h)$.

THEOREM 7.3. If $n \geq 0$, there does not exist a twisted $(4,4)$-product division algebra or a twisted $(8,8)$-product division algebra over $k_{n}=\mathbf{R}\left(\left(T_{1}, \ldots, T_{n}\right)\right)$.

PROOF. By Lemma 7.1, it suffices to show that $H\left(K_{n} / k_{n}\right)=K_{n}^{\times}$for all quadratic extensions $K_{n}$ of $\ell_{n}$. If $n=0, \ell_{0}=\mathbf{R}$ and this is clear. But if $n>0$, then $k_{n}$ has a complete discrete valuation with residue class field $k_{n-1}$. Hence, the assertion follows by induction on $n$ and Lemma 7.2.

We wish next to look at composition algebras and then product algebras over $\ell_{n}$. For this we need some information about quadratic forms over $k_{n}$.

Let

$$
G_{n}=\left\{(-1)^{l_{0}} T_{1}^{l_{1}} T_{2}^{l_{2}} \cdots T_{n}^{l_{n}} \mid l_{i} \in\{0,1\}\right\}
$$

Then, by induction on $n$ and Corollary 1.3 of $[\mathbf{2 1}]$, it follows that $\alpha \mapsto \alpha k_{n}^{\times 2}$ is a bijection of $G_{n}$ onto $\ell_{n}^{\times} / h_{n}^{\times 2}$. We use this bijection to identify $G_{n}=k_{n}^{\times} / h_{n}^{\times 2}$ and hence regard $G_{n}$ as a group. Then, $G_{n} \cong \mathbf{Z}_{2}^{n+1}$ under the mapping $\left(l_{0}, l_{1}, \ldots, l_{n}\right) \mapsto$ $(-1)^{l_{0}} T_{1}^{l_{1}} \cdots T_{n}^{l_{n}}$ of $\mathbf{Z}_{2}^{n+1}$ onto $G_{n}$, where $\mathbf{Z}_{2}$ denotes the integers modulo 2 .

Using the work of Springer on quadratic forms over a field with a complete discrete valuation $[\mathbf{2 1}, \S 6.1]$, the following result is easy to prove: 
PROPOSITION 7.5. Let $q$ be a nondegenerate quadratic form over $k_{n}$. Then

(i) There exist $\delta_{1}, \ldots, \delta_{r} \in G_{n}$ such that

$$
q \cong\left\langle\delta_{1}, \ldots, \delta_{r}\right\rangle \text {. }
$$

(ii) $q$ is anisotropic $\Leftrightarrow \delta_{i} \neq-\delta_{j}$ for all $i, j$.

(iii) If $q$ is anisotropic, the sequence $\delta_{1}, \ldots, \delta_{r}$ is uniquely determined up to order.

(iv) If $q$ is anisotropic and $\delta \in G_{n}$, then $\delta$ is represented by $q \Leftrightarrow \delta=\delta_{i}$ for some $i$.

PROOF. (i) is clear. (ii) follows by induction on $n$ using [21, Proposition 1.9, p. 147]. (iii) follows by induction on $n$ using [21, Corollary 1.6, pp. 145-146]. (iv) follows from (ii) applied to the quadratic form $\left\langle-\delta, \delta_{1}, \ldots, \delta_{r}\right\rangle$.

If $q$ is an anisotropic quadratic form over $h_{n}$, we call the elements $\delta_{1}, \ldots, \delta_{r} \in G_{n}$ satisfying (7.6) the diagonal entries of $q$.

We now consider the composition division algebras over $k_{n}$. These algebras can be described using the methods developed by Petersson in [23]. We indicate here an alternate approach. We need the fact that if

$$
\mathscr{C}=\left(\frac{\alpha_{1}, \ldots, \alpha_{l}}{\ell_{n}}\right),
$$

then $n \leftarrow$ is equivalent to

$$
\begin{gathered}
\langle 1\rangle, \quad\left\langle 1,-\alpha_{1}\right\rangle, \quad\left\langle 1,-\alpha_{1},-\alpha_{2}, \alpha_{1} \alpha_{2}\right\rangle \text { or } \\
\left\langle 1,-\alpha_{1},-\alpha_{2},-\alpha_{3}, \alpha_{1} \alpha_{2}, \alpha_{1} \alpha_{3}, \alpha_{2} \alpha_{3},-\alpha_{1} \alpha_{2} \alpha_{3}\right\rangle
\end{gathered}
$$

according as $l=0,1,2$ or 3 .

If $\mathscr{C}$ is a composition division algebra over $\ell_{n}$, we define $D_{\digamma}$ to be the subgroup of $G_{n}$ consisting of those elements of $G_{n}$ that are represented by the norm $n_{\leftarrow}$ of $\mathscr{C}$. Since $n_{\leftarrow}$ is anisotropic, $D_{\leftarrow}$ is the union of $\{1\}$ and the set of elements of $G_{n}$ that are represented by the pure norm of $\mathscr{C}$ (by Proposition 7.5(iv)). Also, since $\mathscr{C}$ is a division algebra, -1 is not represented by the pure norm of $\mathscr{C}$, and hence $-1 \notin D \leftarrow$. If $\mathscr{C}=\left(\alpha_{1}, \ldots, \alpha_{l}\right)$, then, by Proposition 7.5(iv) and (7.7),

$$
D_{l}=\operatorname{gp}\left(-\alpha_{1}, \ldots,-\alpha_{l}\right),
$$

where $\operatorname{gp}\left(-\alpha_{1}, \ldots,-\alpha_{l}\right)$ denotes the subgroup of $G_{n}$ generated by $\left\{-\alpha_{1}, \ldots,-\alpha_{l}\right\}$, and hence $o\left(D_{f}\right)=2^{p}$, where $p \leq l$.

Proposition 7.9. Suppose $0 \leq l \leq 3$. Suppose $H$ is a subgroup of $G_{n}$ of order $2^{p}$, where $p \leq l$, and suppose that $-1 \notin H$. Then, there exists a unique composition division algebra $\mathscr{C}$ over $k_{n}$ of dimension $2^{l}$ such that $D_{\leftarrow}=H$, namely

$$
\mathscr{C}=\left(\frac{-1, \ldots,-1,-\beta_{1}, \ldots,-\beta_{p}}{\ell_{n}}\right),
$$

where $\beta_{1}, \ldots, \beta_{p}$ is any minimal set of generators of $H$. (Here $p=0$ if $\circ(H)=1$.)

PROOF. Suppose first that $\mathscr{C}$ is given by (7.10). Then, $n_{\varnothing} \cong\left\langle\delta_{1}, \ldots, \delta_{2^{\iota}}\right\rangle$ by (7.7), where $\delta_{1}, \ldots, \delta_{2^{l}}$ are the elements of $H$ repeated $2^{l-p}$ times. Thus, if $\mathscr{C}$ is not a division algebra, then $\delta=-\varepsilon$ for some $\delta, \varepsilon \in H$ (by Proposition 7.5(ii)) and hence $-1 \in H$. Consequently, $\mathscr{C}$ is a division algebra and $D_{\mathscr{C}}=H$. 
Next suppose that $\mathscr{D}=\left(\alpha_{1}, \ldots, \alpha_{l}\right)$ is a division algebra with $D_{\mathscr{D}}=H$. We may assume that the $\alpha_{i}$ 's are arranged so that $H=\operatorname{gp}\left(-\alpha_{1}, \ldots,-\alpha_{p}\right)$. Then, if $p<i \leq l$, we have $-\alpha_{i} \in H$. Consequently, by $(7.7), n_{\mathscr{D}} \cong\left\langle\varepsilon_{1}, \ldots, \varepsilon_{2^{l}}\right\rangle$, where $\varepsilon_{1} \ldots, \varepsilon_{2^{l}}$ are the elements of $H$ repeated $2^{l-p}$ times. Thus, $n_{\mathscr{Z}} \cong n_{\mathscr{C}}$ and so $\mathscr{D} \cong \mathscr{C}[\mathbf{2 4}$, Theorem 3.23].

COROLlary 7.11. Suppose $0 \leq l \leq 3$. Then, the mapping $\mathscr{C} \mapsto D_{\mathscr{C}}$ is a bijection of the set of isomorphism classes of composition division algebras of dimension $2^{l}$ over $k_{n}$ onto the set of subgroups of $G_{n}$ of order $\leq 2^{l}$ that do not contain -1 .

Suppose $n \geq 1$. Then, we may regard $G_{n-1}$ as a subgroup of $G_{n}$ in the obvious fashion. Suppose $0 \leq p \leq 3$. Then, the subgroups of $G_{n}$ of order $2^{p}$ that do not contain -1 are precisely the subgroups:

(i) $H$, where $H$ is a subgroup of order $2^{p}$ of $G_{n-1}$ such that $-1 \notin H$.

(ii) $\operatorname{gp}\left(H, \alpha T_{n}\right)$, where $H$ is a subgroup of order $2^{p-1}$ of $G_{n-1}$ such that $-1 \notin H$ and $\alpha \in G_{n-1}$.

Moreover, subgroups $\operatorname{gp}\left(H, \alpha T_{n}\right)$ and $\operatorname{gp}\left(H^{\prime}, \alpha^{\prime} T_{n}\right)$ as in (ii) are equal if and only if $H=H^{\prime}$ and $\alpha H=\alpha^{\prime} H$.

The remarks in the previous paragraph allow us to inductively construct the subgroups of $G_{n}$ that have order $\leq 2^{3}$ and do not contain -1 . Consequently, by Corollary 7.11 and Proposition 7.9, we can obtain the composition division algebras over $k_{n}$.

If we follow the procedure described above for $n \leq 3$, we obtain Table 7.12 of composition division algebras of dimension $m$ over $k_{3}$ (up to isomorphism). We omit the trivial case $m=1$ from the table. In all cases in the table, the parameters $\rho, \sigma$ and $\tau$ can take on arbitrary values from $\{-1,1\}$.

TABLE 7.12

Subgroups of order $\leq 2^{3}$

of $G_{3}$ not containing -1
Composition division algebras of dimension $m$ over $k_{3}$ (i) $\operatorname{gp}(-\varepsilon), \varepsilon \in G_{3} \backslash\{1\}$

(ii) $\operatorname{gp}\left(-\rho T_{i},-\sigma T_{j}\right)$,

$1 \leq i<j \leq 3$

(iii) $\operatorname{gp}\left(-\rho T_{i},-\sigma T_{j} T_{k}\right), j<k$,

$\{i, j, k\}=\{1,2,3\}$

(iv) $\operatorname{gp}\left(-\rho T_{1} T_{2},-\sigma T_{1} T_{3}\right)$

(v) $\operatorname{gp}\left(-\rho T_{1},-\sigma T_{2},-\tau T_{3}\right)$

$$
\begin{array}{ccc}
m=2 & m=4 & m=8 \\
(\varepsilon) & (-1, \varepsilon) & (-1,-1, \varepsilon) \\
- & \left(\rho T_{i}, \sigma T_{j}\right) & \left(-1, \rho T_{i}, \sigma T_{j}\right) \\
& \left(\rho T_{i}, \sigma T_{j} T_{k}\right) & \left(-1, \rho T_{i}, \sigma T_{j} T_{k}\right) \\
& \left(\rho T_{1} T_{2}, \sigma T_{1} T_{3}\right) & \left(-1, \rho T_{1} T_{2}, \sigma T_{1} T_{3}\right) \\
- & - & \left(\rho T_{1}, \sigma T_{2}, \tau T_{3}\right)
\end{array}
$$

Also, the composition division algebras of dimension $>1$ over $k_{2}$ are the algebras in (i) with $\varepsilon \in G_{2} \backslash\{1\}$ and the algebras in (ii) with $i=1, j=2$. The composition algebras of dimension $>1$ over $h_{1}$ are the algebras in (i) with $\varepsilon \in G_{1} \backslash\{1\}$. Of course, the composition division algebras over $\ell_{0}=\mathbf{R}$ are $\mathbf{R},(-1),(-1,-1)$ and $(-1,-1,-1)$.

Using Table 7.12 (and the remarks below that table), we can next consider all pairs of composition division algebras over $k_{n}, n \leq 3$, at least one of which has dimension 8. For each such pair $\mathscr{C}_{1}, \mathscr{C}_{2}$, we can calculate the Albert form of $\left(\mathscr{C}_{1},-\right) \otimes\left(\mathscr{C}_{2},-\right)$ using (6.3). Then, using Proposition 7.5(ii), we can identify those pairs that give anisotropic Albert forms. The Albert forms so obtained can be compared up to similarity (using Proposition 7.5(iii)). This gives a classification 
of $(8, m)$-product division algebras up to isotopy (by Theorem 6.2) and hence a classification of Lie algebras of index (6.1) over $k_{n}, n \leq 3$ (by Theorems 6.2 and 7.3). In particular, we obtain the following theorem that describes the case $m=8$, $n=3$.

THEOREM 7.13. The division algebra forms of $(8,8)$-product algebras over $k_{3}$ are up to isotopy the algebras

$$
\left(\left(\rho T_{1}, \sigma T_{2}, \tau T_{3}\right),-\right) \otimes((-1,-1, \varepsilon),-),
$$

where $\rho, \sigma, \tau \in\{-1,1\}$ and

$$
\varepsilon \in\left\{-1,-\rho T_{1},-\sigma T_{2},-\tau T_{3}, \rho \sigma T_{1} T_{2}, \rho \tau T_{1} T_{3}, \sigma \tau T_{2} T_{3},-\rho \sigma \tau T_{1} T_{2} T_{3}\right\} .
$$

There are 64 such algebras $(\mathscr{A},-)$ and the corresponding Lie algebras $\mathscr{K}(\mathscr{A},-)$ represent the 64 isomorphism classes of Lie algebras of index $E_{8,1}^{91}$ over $k_{3}$.

ProOF. We need to prove only the first statement. Suppose first that $(\mathscr{A},-)$ is a division algebra form of an $(8,8)$-product algebra over $k_{3}$ with Albert form $Q$. By Theorem $7.3,(\mathscr{A},-)$ is an $(8,8)$-product division algebra. Hence, we may assume that $(\mathscr{A},-)=\left(\mathscr{C}_{1},-\right) \otimes\left(\mathscr{C}_{2},-\right)$, where $\mathscr{C}_{1}, \mathscr{C}_{2}$ are in the last column of Table 7.12. Now if $\mathscr{C}_{1}$ and $\mathscr{C}_{2}$ are both in lines (i)-(iv) of the table, then the pure norms of $\mathscr{C}_{1}$ and $\mathscr{C}_{2}$ both represent 1 and so $Q$ is isotropic. Thus, either $\mathscr{C}_{1}$ or $\mathscr{C}_{2}$ is in line (v). Hence, we may assume that

$$
\mathscr{C}_{1}=\left(\rho T_{1}, \sigma T_{2}, \tau T_{3}\right)
$$

where $\rho, \sigma, \tau \in\{-1,1\}$. Next if $\alpha \neq 1 \in D_{\mathscr{C}_{1}} \cap D_{\mathscr{C}_{2}}$, then $\alpha$ is represented by the pure norms of both $\mathscr{C}_{1}$ and $\mathscr{C}_{2}$ which contradicts the fact that $Q$ is anisotropic. Thus, $D_{\mathscr{\digamma}_{1}} \cap D_{\mathscr{\digamma}_{2}}=\{1\}$. But $o\left(D_{\mathscr{\digamma}_{1}}\right)=8$ and $o\left(G_{3}\right)=16$. Hence, $o\left(D_{\mathscr{C}_{2}}\right) \leq 2$. Thus,

$$
\mathscr{C}_{2}=(-1,-1, \varepsilon)
$$

where $\varepsilon \in G_{3} \backslash\{1\}$. Then, $D_{\mathscr{\leftarrow}_{2}}=\mathrm{gp}(-\varepsilon)$ and since $D_{\mathscr{f}_{1}} \cap D_{\mathscr{C}_{2}}=\{1\}$, we have $-\varepsilon \notin \mathrm{gp}\left(-\rho T_{1},-\sigma T_{2},-\tau T_{3}\right)$, and hence we have (7.15).

Conversely, suppose $(\mathscr{A},-)=\left(\mathscr{C}_{1},-\right) \otimes\left(\mathscr{C}_{2},-\right)$, where $\mathscr{C}_{1}, \mathscr{C}_{2}$ are given by $(7.16)$ and (7.17), and (7.15) is satisfied. Then, using $\lambda=1$ in the definition of the Albert form $Q$ of $(\mathscr{A},-)$, we have (by (6.3)),

$$
\begin{aligned}
Q \cong\left\langle-\rho T_{1},-\sigma T_{2},\right. & -\tau T_{3}, \rho \sigma T_{1} T_{2}, \rho \tau T_{1} T_{3}, \sigma \tau T_{2} T_{3}, \\
& \left.-\rho \sigma \tau T_{1} T_{2} T_{3},-1,-1,-1, \varepsilon, \varepsilon, \varepsilon, \varepsilon\right\rangle .
\end{aligned}
$$

By Proposition 7.5(ii) and (7.15), $Q$ is anisotropic and so $(\mathscr{A},-)$ is a division algebra.

Finally, suppose that $(\mathscr{A},-)$ is given by $(7.14)$, subject to the condition (7.15), suppose that $\left(\mathscr{A}^{\prime},-\right)$ is another such algebra depending on the parameters $\rho^{\prime}, \sigma^{\prime}, \tau^{\prime}$ and $\varepsilon^{\prime}$, and suppose that $\left(\mathscr{A}^{\prime},-\right) \sim(\mathscr{A},-)$. (We again use $\lambda=1$ in the definition of $Q$ and $Q^{\prime}$.) Then, $Q^{\prime} \cong \mu Q$ for some $\mu \in G_{3}$. But -1 is a diagonal entry of $Q$ of multiplicity 3 (by (7.18)). Hence, $-\mu$ is a diagonal entry of $Q^{\prime}$ of multiplicity 3 . Thus, $\mu=1$ (by (7.18) for $Q^{\prime}$ ). Consequently, $Q \cong Q^{\prime}$. Thus, by Proposition 7.5 (iii), $\rho^{\prime}=\rho, \tau^{\prime}=\tau, \sigma^{\prime}=\sigma$ and $\varepsilon^{\prime}=\varepsilon$.

In a similar fashion one obtains a classification up to isotopy of the division algebra forms of $(8, m)$-product algebras over $h_{n}$ for $m=1,2,4$ or 8 and $n \leq$ 
3. Using that classification one obtains the following table giving the number of isomorphism classes of Lie algebras of index $F_{4,1}^{21},{ }^{2} E_{6,1}^{29}, E_{7,1}^{48}$ or $E_{8,1}^{91}$ over $k_{n}$, where $n \leq 3$ :

\begin{tabular}{lrrrr}
$n \backslash I$ & $F_{4,1}^{21}$ & ${ }^{2} E_{6,1}^{29}$ & $E_{7,1}^{48}$ & $E_{8,1}^{91}$ \\
\hline 0 & 1 & 0 & 0 & 0 \\
1 & 3 & 4 & 0 & 0 \\
2 & 11 & 48 & 16 & 0 \\
3 & 51 & 568 & 736 & 64
\end{tabular}

We omit the calculations required to obtain the above table (except for a few remarks below). The interested reader will have no difficulty working out the details using the methods described above.

REMARK. (i) The product algebras giving rise to the diagonal entries in table (7.19) can be easily listed (up to isotopy). Of course the unique $(8,1)$-product division algebra over $k_{0}$ is $((-1,-1,-1),-)$. The four $(8,2)$-product division algebras over $h_{1}$ are

$$
((-1,-1, \varepsilon),-) \otimes\left(\left(\rho T_{1}\right),-\right),
$$

where $\rho \in\{-1,1\}$ and $\varepsilon \in\left\{-1,-\rho T_{1}\right\}$. The sixteen $(8,4)$-product division algebras over $k_{2}$ are

$$
((-1,-1, \varepsilon),-) \otimes\left(\left(\rho T_{1}, \sigma T_{2}\right),-\right),
$$

where $\rho, \sigma \in\{-1,1\}$ and $\varepsilon \in\left\{-1,-\rho T_{1},-\sigma T_{2}, \rho \sigma T_{1} T_{2}\right\}$. We have already, in Theorem 7.13 , listed the sixty-four $(8,8)$-product division algebras over $k_{3}$.

(ii) Petersson [23, p. 421] has given the following expression for the number of isomorphism classes of octonion division algebras (and hence the number of isomorphism classes of Lie algebras of index $F_{4,1}^{21}$ ) over $k_{n}$ :

$$
\frac{1}{21}\left(2^{3 n}+7\left(2^{2 n}+2^{n+1}\right)-1\right)
$$

where $n \geq 0$. This formula can also be used to obtain (and extend) the first column of $(7.19)$.

(iii) We did not find, in our classification of the product division algebras over $\ell_{n}$ for $n \leq 3$, an example of two product division algebras that are isotopic but not isomorphic. If one drops the stipulation that the algebras be division algebras one has such an example. Indeed,

$$
((-1,-1,-1),-) \otimes((-1,-1,-1),-) \sim((1,1,1),-) \otimes((1,1,1),-)
$$

over $\ell_{0}=\mathbf{R}$, since the Albert forms of both sides have maximal Witt index. However, the two algebras are not isomorphic since the factors on the left are division algebras and the factors on the right are split.

\section{REFERENCES}

1. A. A. Albert, On a Wedderburn norm condition for cyclic algebras, Bull. Amer. Math. Soc. 37 (1931), 301-302.

2. A. A. Albert and N. Jacobson, On reduced exceptional simple Jordan algebras, Ann. of Math. (2) 66 (1957), 400417.

3. B. N. Allison, Lie algebras of type $B C_{1}$, Trans. Amer. Math. Soc. 224 (1976), 75-86.

4. $\ldots$, A class of nonassociative algebras with involution containing the class of Jordan algebras, Math. Ann. 237 (1978), 133156. 
5. __ Models of isotropic Lie algebras, Comm. Algebra 7 (1979), 1935-1875.

6. __ Structurable division algebras and relative rank one simple Lie algebras, Proc. 1984 Summer Seminar on Lie Algebras and Related Topics, CMS Conf. Proc., vol. 5, Amer. Math. Soc., Providence, R. I., 1986, pp. 139-156.

7. __ Conjugate inversion and conjugate isotopes of alternative algebras with involution, Algebras, Groups and Geometries 3 (1986), 361-385.

8. B. N. Allison and W. Hein, Isotopes of some nonassociative algebras with involution, J. Algebra 69 (1981), 120142.

9. R. B. Brown, University of Chicago Dissertation, 1964.

10. J. C. Ferrar, Lie algebras of type $E_{6}$, J. Algebra 13 (1969), 57-72.

11. _ Lie algebras of type $E_{6}$. II, J. Algebra 52 (1978), 201-209.

12. W. Hein, A construction of Lie algebras by triple systems, Trans. Amer. Math. Soc. 205 (1975), 79-95.

13. __ On the structure of reduced $\mathcal{J}$-ternary algebras of degree 2, J. Algebra 82 (1983), 157-184.

14. N. Jacobson, Composition algebras and their automorphisms, Rend. Circ. Mat. Palermo 7 (1958), 55-80.

15. _ Structure and representations of Jordan algebras, Amer. Math. Soc. Colloq. Publ., vol. 39, Amer. Math. Soc., Providence, R. I., 1968.

16. __ Lie algebras, Dover, New York, 1979.

17. __ Basic algebra. II, Freeman, San Francisco, Calif., 1980.

18. - Some applications of Jordan norms to involutorial simple associative algebras, Adv. in Math. 48 (1983), 149-165.

19. N. Jacobson and K. McCrimmon, Quadratic Jordan algebras of quadratic forms with base points, J. Indian Math. Soc. 35 (1971), 1-45.

20. I. L. Kantor, Models of exceptional Lie algebras, Soviet Math. Dokl. 14 (1973), 254-258.

21. T. Y. Lam, The algebraic theory of quadratic forms, Benjamin, Reading, Mass., 1973.

22. R. L. Long, Algebraic number theory, Dekker, New York, 1977.

23. H. P. Petersson, Composition algebras over a field with a discrete valuation, J. Algebra 29 (1974), 414-426.

24. R. D. Schafer, Introduction to nonassociative algebras, Academic Press, New York, 1966.

25. __ On structurable algebras, J. Algebra 92 (1985), 400-412.

26. G. B. Seligman, Rational methods in Lie algebras, Dekker, New York, 1966.

27. _ Constructions of Lie algebras and their modules, preprint.

28. J. Tits, Algèbres alternatives, algèbres de Jordan et algèbres de Lie exceptionnelles I. Construction, Nederl. Akad. Wetensch. Proc. Ser. A 69 (1966), 223-237.

29. __ Classification of algebraic semisimple groups, Proc. Sympos. Pure Math., vol. 9, Amer. Math. Soc., Providence, R. I., 1966, pp. 33-62.

30. __ Représentations linéaires irréductiblès d'un group réductif sur un corps quelconque, J. Reine Angew. Math. 247 (1971), 196-220.

31. M. L. Tomber, Lie algebras of type F, Proc. Amer. Math. Soc. 4 (1953), 759-768.

Department of Mathematics, University of Alberta, Edmonton, Alberta, CANADA T6G 2G1 